\title{
Produit Croisé d'une Algèbre de von Neumann par Une Algèbre de Kac, II
}

\author{
par \\ Michel EnocK* et Jean-Marie Schwartz*
}

\section{Introduction}

La construction du produit croisé d'une algèbre de von Neumann par un groupe continu d'automorphismes est à l'origine des résultats récents [2], [20]) dans l'étude de la structure des facteurs.

Soit $G$ un groupe localement compact. M. Landstad a remarqué dans [12] l'intérêt des propriétés d'algèbre de Hopf-von Neumann de $L^{\infty}(G)$ et $\mathscr{M}(G)$ (respectivement algèbre des fonctions mesurables essentiellement bornées sur $G$ et algèbre de la représentation régulière gauche de $G$ ) pour traiter la construction des produits croisés. La construction qu'il utilise s'interprète en fait comme une "action" de $\mathscr{M}(G)$ (considéré comme substitut-dans le cas non-abélien - du groupe dual de $G$ ) sur une algèbre de von Neumann.

Les algèbres de Kac ([5], [17]) dont $L^{\infty}(G)$ et $\mathscr{M}(G)$ sont des cas particuliers duaux l'un de l'autre, apparaissent alors comme devant fournir un cadre bien adapté à une généralisation de la notion de produit croisé. Celle-ci contenant à la fois la construction du produit croisé par un groupe [20], et par un "dual de groupe" [13], [14].

Une première partie de ce travail a déjà été faite [4], mais elle ne fournit pas tous les résultats dont on pouvait espérer la généralisation, notamment en ce qui concerne la dualité. Les obstacles techniques étant désormais levés, nous donnons ci-après une théorie plus complète, qui permet de généraliser au cas des algèbres de Kac le théorème de dualité de $\mathrm{M}$. Takesaki [20], le thèoréme de commutation de T. Digernes [3] et la caractérisation des produits croisés, due à M. Landstad [12].

Communiqué par H. Araki, le 20, février 1978.

* Université Pierre et Marie Curie (U.E.R. 48), Laboratoire de Mathématiques Fondamentales, 4, place Jussieu, 75230 Paris Cedex 05, France. 
Dans le cas particulier des "duaux de groupe" (c'est-à-dire, dans notre terminologie, des algèbres de Kac symétriques) ces résultats ont été démontrés indépendamment dans [13], [14], [19].

Nous avons pu également amorcer une théorie du poids dual sur le produit croisé d'une algèbre de von Neumann par une algèbre de Kac qui étend partiellement les résultats de [20], [3], [16] et [10], et fait apparaître en particulier l'intérêt de la notion "d'invariance relative" d'un poids par rapport à une action, qui caractérise les poids duaux.

Tous ces résultats ont été annoncés dans [7]. Ils ont été exposés par le premier co-auteur aux congrès internationaux de Marseille (Juin 1977) et Leipzig (Septembre 1977). La mise au point définitive de la rédaction a été volontairement retardée pour pouvoir se référer à la deuxième édition de [17].

\section{Prolègoménes}

(P1) Soit $A$ une algèbre de von Neumann opérant sur un espace hilbertien $K$. On notera $P(A)$ l'ensemble des poids normaux, semi-finis, fidèles sur $A$. Certaines des constructions concernant ceux-ci se généralisent aux poids non fidèles; il suffit de remarquer que si $\Psi$ est un poids normal semi-fini sur $A$ et $P$ son support ([1], p. 95), la restriction de $\Psi$ à l'algèbre réduite $A_{P}$, que l'on notera $\Psi_{P}$, appartient à $P\left(A_{P}\right)$.

Réciproquement, soient $P$ un projecteur de $A$ et $\Psi$ un élément de $P\left(A_{P}\right)$, l'application définie pour tout $x$ de $A^{+}$par

$$
\Psi^{P}(x)=\Psi\left(x_{P}\right)
$$

où $x_{P}$ désigne la restriction de $P x$ à $P K$, est un poids normal semi-fini sur $A$, de support $P$.

On a clairement $\left(\Psi_{P}\right)^{P}=\Psi$ pour tout poids normal semi-fini sur $A$, de support $P$, et $\left(\Psi^{P}\right)_{P}=\Psi$ pour tout $\Psi$ de $P\left(A_{P}\right)$.

(P2) Soient $h$ un opérateur autoadjoint positif, affilié au centre de $A, \Psi$ un poids normal semi-fini sur $A$ de support $P$. Notons $h_{P}$ la restriction de $P h$ à $P K$. On pose: $\quad \Psi(h)=\left(\Psi_{P}\left(h_{P} \cdot\right)\right)^{P}$

où $\Psi_{P}\left(h_{P}.\right)$ a été défini en [15], Section 4.Cette définition coincide avec celle donnée en [15], Section 4, où, l'hypothèse de fidélité qui ne joue en fait aucun rôle peut être supprimée. 
Remarquons que pour $\xi$ dans le domaine de $h^{1 / 2}$, on a

$$
\omega_{\xi}\left(h_{.}\right)=\omega_{h^{1 / 2 \xi}}
$$

où $\omega_{\xi}$ est la forme vectorielle associée à $\xi$.

On peut démontrer, comme en [15], Section 4.3 que si $h$ et $k$ sont deux opérateurs autoadjoints positifs affiliés au centre de $A$, si $\Psi^{\prime}$ désigne le poids $\Psi(h$.$) , on a \psi^{\prime}(k)=.\psi(\overline{h k}$.$) , où \overline{h k}$ désigne la fermeture de l'opérateur $h k$. En particulier, si $h$ est non singulier, on a $\psi=\psi^{\prime}\left(h^{-1}\right.$.).

(P3) Soit $\left(h_{i}\right)_{i \in I}$ une famille d'éléments positifs du centre de $A$, telle que $h_{i} T h$ au sens de [15], 4.1. Alors, en conservant les notations précédentes, si $x$ appartient à $\mathfrak{M}_{\Psi}, h_{i} x$ appartient à $\mathfrak{M}_{\Psi}$ pour tout $i$ de $I$, et $\lim _{i} \Psi\left(h_{i} x\right)=\Psi^{\prime}(x)$.

En particulier, pour tout $\varepsilon$, réel positif, on posera $h_{\varepsilon}=h(1+\varepsilon h)^{-1},\left(h_{\varepsilon}\right)_{\varepsilon}>0$ est alors une famille d'éléments positifs du centre de $A$ telle que $h_{\varepsilon} \nearrow h$ (voir [15], 4.1).

$(\mathbb{P} 4)$ Soit $\Psi_{i}$ un poids normal semi-fini sur une algèbre de von Neumann $A_{i}$, de support $P_{i}(i=1,2)$. On pose:

$$
\Psi_{1} \otimes \Psi_{2}=\left(\Psi_{1_{P_{1}}} \otimes \Psi_{2_{P_{2}}}\right)^{p_{1} \otimes P_{2}}
$$

où le produit tensoriel $\Psi_{1_{P_{1}}} \otimes \Psi_{2_{P_{2}}}$ a été défini en [2], 1.1.2.

Soit alors $h_{i}$ un opérateur autoadjoint positif affilié au centre de $A_{i}$ $(i=1,2)$. On a clairement:

$$
\Psi_{1}\left(h_{1} \cdot\right) \otimes \Psi_{2}\left(h_{2} .\right)=\left(\Psi_{1} \otimes \Psi_{2}\right)\left(\left(h_{1} \otimes h_{2}\right) .\right)
$$

où $h_{1} \otimes h_{2}$ désigne la fermeture du produit tensoriel algébrique des applications linéaires $h_{1}$ et $h_{2}$, (en particulier, pour tout $t$ de $R$ on a $\left.\left(h_{1} \otimes h_{2}\right)^{i t}=h_{1}^{i t} \otimes h_{2}^{2 t}\right)$. En effet, si $\Psi_{1}$ et $\Psi_{2}$ sont fidèles, cela résulte de [2], 1.2.3. (b) et [11] 1.3.2., et la transcription au cas non fidèle se fait sans difficulté.

(P5) On notera $\operatorname{ext}^{+} A$ l'extension positive de $A$, définie et notée $\hat{A}_{+}$dans [9], dont on utilisera toutes les autres notations. En particulier, si $\Psi$ est un poids normal sur $A$, on notera encore $\Psi$ son extension canonique à $\operatorname{ext}^{+} A$ ([9], Prop. 1.10).

\section{Préliminaires concernant les algebres de Kac}

Dans tout ce chapitre, $\mathbb{K}=(M, \Gamma, \kappa, \varphi)$ désigne une algèbre de Kac (voir 
[5], Déf. 1.3.1 et [17], Th. III. 34.) On utilise les notations de [5] et [17]. En particulier $W$ désigne l'opérateur (unitaire) fondamental associé à $\boldsymbol{K}$ et $H$ l'espace hilbertien $H_{\varphi}$ (voir le formulaire de [5], 4.3.9.).

D'autre part, $A$ désignera une algèbre de von Neumann, $\Psi$ un élément de $P(A)$ (voir (P1)). On notera indifféremment $i$ l'identité de $A$ et de $M$.

Lemma 1.1. $W(1 \otimes \hat{\Delta}) W^{*}=\hat{\Delta} \otimes \hat{\Delta}$.

Démonstration: Cela résulte de [17], III. 38 (ii) appliqué à $\boldsymbol{K}^{\wedge}$, car on a $\hat{W}=\sigma W^{*} \sigma$, d'après [5], 4.1.4. (a).

Corollaire 1.2: Soit $\varepsilon$ un réel positif. On a:

(i) $\Gamma\left(\hat{\Delta}_{\mathrm{g}}\right)=(\hat{\Delta} \otimes \hat{\Delta})_{\mathrm{g}}$

(ii) $\Gamma\left(\left(\hat{\Lambda}^{1 / 2}\right)_{\varepsilon}\right)=\left(\hat{\Lambda}^{1 / 2} \otimes \hat{\Lambda}^{1 / 2}\right)_{8}$

(iii) $\Gamma\left(\left(\hat{\Delta}^{-1 / 2}\right)_{\varepsilon}\right)=\left(\hat{\Delta}^{-1 / 2} \otimes \hat{\Lambda}^{-1 / 2}\right)_{\varepsilon}$

avec les notations introduites en ( $\mathrm{P} 3)$.

Démonstration: On a:

$$
\begin{aligned}
W(1 \otimes(1+\varepsilon \hat{\Delta})) W^{*} & =1 \otimes 1+\varepsilon W(1 \otimes \hat{\Delta}) W^{*} \\
& =1 \otimes 1+\varepsilon(\hat{\Delta} \otimes \hat{\Delta}) \text { d'après I.1. }
\end{aligned}
$$

d'où :

$$
W\left(1 \otimes(1+\varepsilon \hat{\Delta})^{-1}\right) W^{*}=\left(1 \otimes 1+\varepsilon\left(\hat{\Delta} \otimes \hat{\Delta}^{-1}\right)\right)
$$

puis, comme d'après [5], 2.2.5.(b), on a

$$
\Gamma\left(\hat{\Delta}_{\varepsilon}\right)=W\left(1 \otimes \hat{\Delta}(1+\varepsilon \hat{\Delta})^{-1}\right) W^{*}
$$

on trouve:

$$
\begin{aligned}
\Gamma\left(\hat{\Delta}_{\varepsilon}\right) & \supset W(1 \otimes \hat{\Delta}) W^{*} W\left(1 \otimes(1+\varepsilon \hat{\Delta})^{-1}\right) W^{*} \\
& =(\hat{\Delta} \otimes \hat{\Delta})(1 \otimes 1+\varepsilon(\hat{\Delta} \otimes \hat{\Delta}))^{-1} \text { d'apès I.1. et ce qui précède } \\
& =(\hat{\Delta} \otimes \hat{\Delta})_{\mathrm{\varepsilon}}
\end{aligned}
$$

d'où (i), les deux membres étant bornés. Les démonstrations de (ii) et (iii) sont identiques.

Proposition 1.3: Pour tout $x$ de $M^{+}$, on a:

$$
(\varphi \otimes i)(\Gamma(x))=\varphi(x) \cdot \hat{\Delta}^{-1}
$$

Démonstration: Soit $\omega$ dans $M_{*}^{+}$. On pose $\omega^{\prime}=\omega\left(\hat{\Lambda}^{-1}\right.$.) (voir (P2)). Grâce à (P2), (P4) et [5], 4.2.1. on a alors: 


$$
\varphi \otimes \omega=\left(\varphi \circ \kappa \otimes \omega^{\prime}\right)((\hat{\Delta} \otimes \hat{\Delta}) .) .
$$

Donc, pour tout $x$ de $M^{+}$, on a, d'après (P3):

$$
\begin{aligned}
(\varphi \otimes \omega)(\Gamma(x)) & =\lim _{\varepsilon \rightarrow 0}\left(\varphi \circ \kappa \otimes \omega^{\prime}\right)\left((\hat{\Delta} \otimes \hat{\Delta})_{\varepsilon} \Gamma(x)\right) \\
& =\lim _{\varepsilon \rightarrow 0}\left(\varphi \circ \kappa \otimes \omega^{\prime}\right)\left(\Gamma\left(\hat{\Delta}_{\varepsilon} x\right)\right) \text { d'après I.2.(i). }
\end{aligned}
$$

Or, l'axiome (NKiii) de [17], th.III.34, appliqué à l'algèbre de Kac réfléchie $\boldsymbol{K}^{\text {s }}$ (cf [17], Prop VII.6.), donne, pour tout $y$ de $M^{+}$:

$$
(i \otimes \varphi \circ \kappa)(\varsigma \Gamma(y))=(\varphi \circ \kappa \otimes i)(\Gamma(y))=\varphi \circ \kappa(y) .
$$

Ainsi, on obtient:

$$
\begin{aligned}
(\varphi \otimes \omega)(\Gamma(x)) & =\lim _{\varepsilon \rightarrow 0} \varphi \circ \kappa\left(\hat{\Delta}_{\mathrm{\varepsilon}} x\right) \omega^{\prime}(1) \\
& =\varphi(x) \omega\left(\hat{\Delta}^{-1}\right) \text { d'après [5], 4.2.1. }
\end{aligned}
$$

d'où le résultat, par définition de ext ${ }^{+} M$.

Corollaire 1.4: Soient $x$ dans $(A \otimes M)^{+}$et $\xi$ dans $\mathscr{D}\left(\hat{\Delta}^{-1 / 2}\right)$. On a:

$$
\left(\psi \otimes \varphi \otimes \omega_{\xi}\right)((i \otimes \Gamma)(x))=(\psi \otimes \varphi)(x)\left\|\hat{\Delta}^{-1 / 2} \xi\right\|^{2} .
$$

Démonstration: Soient $x_{1}$ dans $A^{+}, x_{2}$ dans $M^{+}$. On a

$$
\begin{aligned}
\left(\psi \otimes \varphi \otimes \omega_{\xi}\right)\left((i \otimes \Gamma)\left(x_{1} \otimes x_{2}\right)\right) & =\psi\left(x_{1}\right)\left(\varphi \otimes \omega_{\xi}\right)\left(\Gamma\left(x_{2}\right)\right) \\
& =\psi\left(x_{1}\right) \varphi\left(x_{2}\right)\left\|\hat{\Delta}^{-1 / 2} \xi\right\|^{2} \text { d'après } \\
& =(\psi \otimes \varphi)\left(x_{1} \otimes x_{2}\right)\left\|\hat{\Delta}^{-1 / 2} \xi\right\|^{2}
\end{aligned}
$$

I.4 et [17], 0.4 .

Considérons sur $A \otimes M$ les deux poids $\Phi$ et $\Psi$ définis par

$$
\begin{aligned}
& \Phi(x)=(\psi \otimes \varphi)(x)\left\|\hat{\Delta}^{-1 / 2} \xi\right\|^{2} \\
& \Psi(x)=\left(\psi \otimes \varphi \otimes \omega_{\xi}\right)((i \otimes \Gamma)(x)) .
\end{aligned}
$$

Le poids $\Phi$ est normal, semi-fini et fidèle et $\sigma_{t}^{\Phi}=\sigma_{t}^{\psi} \otimes \sigma_{t}^{\varphi}$ pour tout $t$ de $\boldsymbol{R}$. Le poids $\Psi$ est normal et semi-fini (d'après le calcul précédent). De plus:

$$
\begin{aligned}
\Psi\left(\sigma_{t}^{\Phi}(x)\right) & =\left(\psi \otimes \varphi \otimes \omega_{\xi}\right)\left((i \otimes \Gamma)\left(\sigma_{t}^{\psi} \otimes \sigma_{t}^{\varphi}\right)(x)\right) \\
& =\left(\psi \otimes \varphi \otimes \omega_{\xi}\right)\left(\left(\sigma_{t}^{\psi} \otimes \sigma_{t}^{\varphi} \otimes i\right)(i \otimes \Gamma)(x)\right) \text { d'après [5], 4.2.5 (a) } \\
& =\psi(x) .
\end{aligned}
$$

En appliquant par exemple [5], lemme 4.1.1., on en déduit $\Phi=\Psi$.

Lemme I.5: En notant indifféremment 5 l'isomorphisme de $M \otimes A$ sur 
$A \otimes M$ défini par $\varsigma(x \otimes y)=y \otimes x$, ou son inverse, les morphismes suivants, définis sur $M \otimes A:(\varsigma \otimes i)(i \otimes \varsigma)(\Gamma \otimes i)$ et $(i \otimes \Gamma) \varsigma$ sont égaux.

Démonstration: Soient $a$ et $b$ dans $M$ et $c$ dans $A$. On $a$ :

$$
(\varsigma \otimes i)(i \otimes \varsigma)(a \otimes b \otimes c)=c \otimes a \otimes b
$$

par linéarité et continuité, on en déduit

$$
(\varsigma \otimes i)(i \otimes \varsigma)(\Gamma(a) \otimes c)=c \otimes \Gamma(a)
$$

ou encore $(\varsigma \otimes i)(i \otimes \varsigma)(\Gamma \otimes i)(\alpha \otimes c)=(i \otimes \Gamma) \varsigma(\alpha \otimes c)$ d'où le résultat par linéarité et continuité.

Lemme 1.6: Soit $x$ dans $(M \otimes A)^{+}$. On a:

$$
(i \otimes \varphi \otimes i)((\Gamma \otimes i)(x))=1 \otimes(\varphi \otimes i)(x) .
$$

Démonstration: Cela résulte de l'axiome (NKiii) de [17], th.III.34. et de la définition des produits tensoriels de poids opératoriels, normaux, semifinis, fidèles ([9], 5.6).

Lemme 1.7: Soient $x$ dans $\mathfrak{N}_{\varphi}$, a dans $\mathfrak{N}_{\varphi \otimes i}$. Alors, $(\Gamma \otimes i)(a)(x \otimes 1 \otimes 1)$ appartient à $\mathfrak{\Re}_{\varphi \otimes \varphi \otimes i}$, et pour tout $\Omega$ de $A_{*}^{+}$, on $a$ :

$$
\Lambda_{\varphi \otimes \varphi \otimes \Omega}((\Gamma \otimes i)(a)(x \otimes 1 \otimes 1))=(W \otimes 1) \Lambda_{\varphi \otimes \varphi \otimes \Omega}(x \otimes a) .
$$

Démonstration: Soient $a_{1}$ dans $\mathfrak{N}_{\varphi}, a_{2}$ dans $A$. On a:

$$
\begin{aligned}
(\Gamma \otimes i)\left(a_{1} \otimes a_{2}\right)(x \otimes 1 \otimes 1) & =\left(\Gamma\left(a_{1}\right) \otimes a_{2}\right)(x \otimes 1 \otimes 1) \\
& \left.=\left(\Gamma\left(a_{1}\right)\right)(x \otimes 1)\right) \otimes a_{2} .
\end{aligned}
$$

Grâce à l'axiome (Kiii) de [5], 1.3.1., on en déduit que

$$
(\Gamma \otimes i)\left(a_{1} \otimes a_{2}\right)(x \otimes 1 \otimes 1) \in \mathfrak{N}_{\varphi \otimes \varphi \otimes i}
$$

De plus, d'après [5]. Proposition 2.1.1., on a:

$$
\begin{aligned}
\Lambda_{\varphi \otimes \varphi \otimes \Omega}\left((\Gamma \otimes i)\left(a_{1} \otimes a_{2}\right)(x \otimes 1 \otimes 1)\right) & =\Lambda_{\varphi \otimes \varphi}\left(\Gamma\left(a_{1}\right)(x \otimes 1)\right) \otimes \Lambda_{\Omega}\left(a_{2}\right) \\
& =W\left(\Lambda_{\varphi}(x) \otimes \Lambda_{\varphi}\left(a_{1}\right)\right) \otimes \Lambda_{\Omega}\left(a_{2}\right) .
\end{aligned}
$$

Si $a_{2}$ appartient à $\mathfrak{R}_{\psi}$, on montre de même que $(\Gamma \otimes i)\left(a_{1} \otimes a_{2}\right)(x \otimes 1 \otimes 1)$ appartient à $\mathfrak{R}_{\varphi \otimes \varphi \otimes \psi}$ et que

$$
\Lambda_{\varphi \otimes \varphi \otimes \psi}\left((\Gamma \otimes i)\left(a_{1} \otimes a_{2}\right)(x \otimes 1 \otimes 1)\right)=W\left(\Lambda_{\varphi}(x) \otimes \Lambda_{\varphi}\left(a_{1}\right)\right) \otimes \Lambda_{\psi}\left(a_{2}\right) .
$$

Considérons alors sur $M \otimes A$ les deux poids $\Phi$ et $\Psi$ définis par: 


$$
\begin{aligned}
& \Phi(b)=\varphi\left(x^{*} x\right)(\varphi \otimes \psi)(b) \\
& \Psi(b)=(\varphi \otimes \varphi \otimes \psi)\left(\left(x^{*} \otimes 1 \otimes 1\right)(\Gamma \otimes i)(b)(x \otimes 1 \otimes 1)\right)
\end{aligned}
$$

pour $b$ dans $(M \otimes A)^{+}$.

Le poids $\Phi$ est normal, semi-fini, fidèle; le poids $\Psi$ est normal et semifini d'après ce qui précède. Comme en $\mathrm{I} .4$ on montre que $\Psi$ est invariant par $\sigma_{t}^{\Phi}$, puis, en utilisant ce qui précède que $\Phi=\Psi$.

On en déduit alors que le poids opératoriel normal de $M \otimes A \operatorname{sur} A$ défini par $b \mapsto(\varphi \otimes \varphi \otimes i)\left(\left(x^{*} \otimes 1 \otimes 1\right)(\Gamma \otimes i)(b)(x \otimes 1 \otimes 1)\right)$ est semi-fini et fidèle ([9], lemme 2.6.) et qu'il est égal au poids opératoriel semi-fini et fidèle défini par $b \mapsto \varphi\left(x \varphi^{*} x\right)(\varphi \otimes i)(b)([9]$, lemme 4.8.).

Il en résulte que:

$(\varphi \otimes \varphi \otimes \Omega)\left(\left(x^{*} \otimes 1 \otimes 1\right)(\Gamma \otimes i)\left(a^{*} a\right)(x \otimes 1 \otimes 1)\right)=\varphi\left(x^{*} x\right)(\varphi \otimes \Omega)\left(a^{*} a\right)$.

Après polarisation, on en déduit l'existence d'une isométrie de $H_{\varphi \otimes \varphi \otimes \Omega}$ qui envoie $\Lambda_{\varphi \otimes \varphi \otimes \Omega}(x \otimes a)$ sur $\Lambda_{\varphi \otimes \varphi \otimes \Omega}((\Gamma \otimes i)(\alpha)(x \otimes 1 \otimes 1))$.

Il résulte de $(*)$ que cette isométrie coïncide avec $W \otimes 1$ sur les éléments de la forme $\Lambda_{\varphi \otimes \varphi \otimes \Omega}\left(x \otimes a_{1} \otimes a_{2}\right)$; donc, par linéarité et continuité, elle est égale à $W \otimes 1$, et le lemme en résulte.

Lemme 1.8: Soient $x, y$ dans $\mathfrak{N}_{\varphi \otimes_{i}}, \omega$ dans $M_{*}^{+}$et $\theta$ dans $A_{*}^{+}$. On $\alpha$ :

(i) $(\omega \otimes \varphi \otimes \theta)\left((\Gamma \otimes i)\left(y^{*}\right)(1 \otimes x)\right)=\left\langle W^{*} \otimes 1, \omega \otimes \Omega_{\Lambda \varphi \otimes \theta}(x), \Lambda_{\varphi \otimes \theta}(y)\right\rangle$

(ii) $(\omega \otimes \varphi \otimes \theta)\left(\left(1 \otimes y^{*}\right)(\Gamma \otimes i)(x)\right)=\left\langle W \otimes 1, \omega \otimes \Omega_{\Lambda_{\varphi \otimes \theta}(x), \Lambda_{\varphi \otimes \theta}(y)}\right\rangle$

(il rséulte du lemme I.6. que les premiersme mbres de ces égalités ont un sens).

Démonstration: Soit $\xi$ dans $\mathscr{2}$. On a:

$$
\begin{aligned}
& \left(\omega_{\xi} \otimes \varphi \otimes \theta\right)\left((\Gamma \otimes i)\left(y^{*}\right)(1 \otimes x)\right)=(\varphi \otimes \varphi \otimes \theta)\left((\pi(\xi) * \otimes 1 \otimes 1)(\Gamma \otimes i)\left(y^{*}\right)\right. \\
& (\pi(\xi) \otimes x)) \quad \text { d'après [17], II.11. } \\
& =\left(\Lambda_{\varphi \otimes \varphi \otimes \theta}(\pi(\xi) \otimes x) \mid \Lambda_{\varphi \otimes \varphi \otimes \theta}((\Gamma \otimes i)(y)(\pi(\xi) \otimes 1 \otimes 1)) \quad\right. \text { d'après I.7. } \\
& =\left(\xi \otimes \Lambda_{\varphi \otimes \theta}(x) \mid(W \otimes 1)\left(\xi \otimes \Lambda_{\varphi \otimes \theta}(y)\right)\right) \quad \text { d'après I.7. } \\
& =\left\langle W^{*} \otimes 1, \omega_{\xi} \otimes \Omega_{\Lambda_{\varphi \otimes \theta}(x), \Lambda_{\varphi \otimes \theta}(y)}\right\rangle \text {. }
\end{aligned}
$$

Il résulte du lemme I.6. que $(\Gamma \otimes i)\left(y^{*}\right)(1 \otimes x)$ appartient à $\mathfrak{M}_{i \otimes \varphi \otimes \theta}$, cette égalité peut donc s'écrire:

$$
\left\langle(i \otimes \varphi \otimes \theta)\left((\Gamma \otimes i)\left(y^{*}\right)(1 \otimes x)\right), \omega_{\xi}\right\rangle=\left\langle W^{*} \otimes 1, \omega_{\xi} \otimes \Omega_{\Lambda_{\varphi \otimes \theta}}(x), \Lambda_{\varphi \otimes \theta}(y)\right\rangle
$$

d'où (i), par continuité et densité des $\omega_{\xi}$ dans $M_{*}^{+}$. De plus on a: 


$$
\begin{array}{rlrl}
\left\langle W \otimes 1, \omega \otimes \Omega_{\Lambda_{\varphi \otimes \theta}(x), \Lambda_{\varphi \otimes \theta}(y)}\right\rangle & =\left\langle W^{*} \otimes 1, \omega \otimes \Omega_{\Lambda_{\varphi \otimes \theta}(y), \Lambda_{\varphi \otimes \theta}(x)}\right\rangle^{-} & \\
& =(\omega \otimes \varphi \otimes \theta)\left((\Gamma \otimes i)\left(x^{*}\right)(1 \otimes y)\right)^{-} & & \text {d'après (i) } \\
& =(\omega \otimes \varphi \otimes \theta)\left(\left(1 \otimes y^{*}\right)(\Gamma \otimes i)(x)\right), & & \text { d'où (ii). }
\end{array}
$$

Proposition 1.9: $\quad$ Soient $x, y$ dans $\mathfrak{N}_{\varphi \otimes_{i}}$. On $a$

$(i \otimes \varphi \otimes i)\left[(\Gamma \otimes i)\left(y^{*}\right)(1 \otimes x)\right]=(\kappa \otimes i)\left[(i \otimes \varphi \otimes i)\left(\left(1 \otimes y^{*}\right)(\Gamma \otimes i)(x)\right)\right]$

(Il résulte de I.6. que les deux termes ont un sens).

Démonstration: Soient $\omega$ dans $M_{*}^{+}$et $\theta$ dans $A_{*}^{+}$. On a:

$$
\begin{aligned}
&(\omega \otimes \varphi \otimes \theta)\left((\Gamma \otimes i)\left(y^{*}\right)(1 \otimes x)\right)=\left\langle W \otimes 1, \omega \otimes \Omega_{\Lambda_{\varphi \otimes \theta}(x), \Lambda_{\varphi \otimes \theta}(y)}{ }^{*}\right. \\
& \text { d'après I.8. (i). } \\
&=\left\langle(\kappa \otimes i)(W) \otimes 1, \omega \otimes \Omega_{\Lambda \varphi \otimes \theta}(x), \Lambda_{\varphi \otimes \theta}(y)\right. \\
& \text { d'après [7], lemme I.13. } \\
&=\left\langle W \otimes 1, \omega \circ \kappa \otimes \Omega_{\Lambda_{\varphi} \otimes \theta}(x), \Lambda_{\varphi \otimes \theta}(y)\right\rangle \\
&=(\omega \circ \kappa \otimes \varphi \otimes \theta)\left(\left(1 \otimes y^{*}\right)(\Gamma \otimes i)(x)\right) \\
& \text { d'après I.8. (ii) }
\end{aligned}
$$

d'où le résultat.

Corollaire 1.10: Soient $x$ et $y$ dans $\mathfrak{N}_{i \otimes \varphi_{\kappa}}$. Alors, les deux expressions $(i \otimes \varphi \circ \kappa \otimes i)\left((i \otimes \Gamma)(y)^{*}(x \otimes 1)\right)$ et $(i \otimes \kappa)(i \otimes \varphi \circ \kappa \otimes i)\left(\left(y^{*} \otimes 1\right)(i \otimes \Gamma)(x)\right)$ ont un sens et sont égales.

Démonstration: Appliquons la proposition I.9. à l'algèbre de Kac réfléchie $\boldsymbol{K}^{\mathrm{s}}$ ([17], Prop. VII.6.). On trouve, pour $a, b$ dans $\mathfrak{N}_{\varphi_{\circ} \otimes_{i}}$ :

$(i \otimes \varphi \circ \kappa \otimes i)\left((\varsigma \Gamma \otimes i)\left(b^{*}\right)(1 \otimes a)\right)=(\kappa \otimes i)\left((i \otimes \varphi \circ \kappa \otimes i)\left(1 \otimes b^{*}\right)(\varsigma \Gamma \otimes i)(a)\right)$

En appliquant $\varsigma$ aux deux membres, on obtient:

$$
\begin{aligned}
& (i \otimes \varphi \circ \kappa \otimes i)\left((\varsigma \otimes i)(i \otimes \varsigma)(\varsigma \otimes i)(\Gamma \otimes i)\left(b^{*}\right)(1 \otimes a)\right)= \\
& \quad=(i \otimes \kappa)\left((i \otimes \varphi \circ \kappa \otimes i)(\varsigma \otimes i)(i \otimes \varsigma)(\varsigma \otimes i)(1 \otimes b)^{*}(\varsigma \Gamma \otimes i)(a)\right)
\end{aligned}
$$

soit,

$(i \otimes \varphi \circ \kappa \otimes i)\left((\varsigma \otimes i)(i \otimes \varsigma)(\Gamma \otimes i)\left(b^{*}\right)(\varsigma a \otimes 1)\right)=$

$$
=(i \otimes \kappa)\left((i \otimes \varphi \circ \kappa \otimes i)\left(\varsigma b^{*} \otimes 1\right)(\varsigma \otimes i)(i \otimes \varsigma)(\Gamma \otimes i)(a)\right)
$$

d'où, grâce au lemme I.5: 
$(i \otimes \varphi \circ \kappa \otimes i)\left((\Gamma \otimes i)\left(\varsigma b^{*}\right)(\varsigma a \otimes 1)\right)=(i \otimes \kappa)(i \otimes \varphi \circ \kappa \otimes i)\left(\left(\varsigma b_{*} \otimes 1\right)(\Gamma \otimes i)(\varsigma a)\right)$.

Rappel 1.11: Il résulte de [5], 2.2.4.1., 3.1.5.(a) et 1.2.2.1. que, pour tous $\xi, \eta$, dans $H$, on a $\omega_{\xi, \eta} \circ \kappa=\omega \hat{\jmath}_{\eta}, \hat{\jmath}_{\xi}$.

Lemme 1.12: Soit $\xi$ dans $\mathscr{D}\left(\hat{\Delta}^{-1 / 4}\right)$. On $a \omega_{\hat{\Delta}^{-1 / 4}} \circ \kappa=\left(\omega_{\xi} \circ \kappa\right)\left(\hat{\Delta}^{-1 / 2}.\right)$.

Démonstration: On a: $\omega_{\hat{A}^{-1 / 4}} \circ \kappa=\omega_{\hat{A}} \hat{A}^{-1 / 4} \xi$ d'après $\mathrm{I} .11$

$$
\begin{aligned}
& =\omega_{\hat{\Delta}^{1 / 4} \hat{\jmath}_{\xi}} \\
& =\omega_{\xi}\left(\hat{\Delta}^{1 / 2} \cdot\right) \quad \text { voir }(\mathrm{P} 2) \\
& =\omega_{\xi} \circ \kappa\left(\hat{\Delta}^{11 / 2} \cdot\right) \quad \mathrm{d}^{\prime} \text { après } \mathbb{I} .11 .
\end{aligned}
$$

Corollaire 1.13: Soient $x$ et $y$ dans $\mathfrak{\Re}_{\psi \otimes \varphi}, \xi$ dans $\left.\mathscr{D}\left(\hat{\Delta}^{1 / 4}\right) \cap \mathscr{D} \hat{\Delta}^{-1 / 4}\right)$. Alors, les deux expressions $\left(\psi \otimes \varphi \otimes \omega_{\hat{\Delta}^{1 / 4} \xi}\right)\left((i \otimes \Gamma)\left(y^{*}\right)(x \otimes 1)\right)$ et $\left(\psi \otimes \varphi \otimes \omega_{\hat{\Delta}^{-1 / 4}} \circ \kappa\right)$ $\left(\left(y^{*} \otimes 1\right)(i \otimes \Gamma)(x)\right)$ ont un sens et sont égales.

Démonstration: Soit $z$ dans $(A \otimes M)^{+}$. Grâce au corollaire I.4, on a:

$$
\left(\psi \otimes \varphi \otimes \omega_{\hat{\Delta}^{1 / 4}}\right)((i \otimes \Gamma)(z))=(\psi \otimes \varphi)(z)\left\|\hat{\Delta}^{-1 / 4} \xi\right\|^{2} .
$$

Donc, $(i \otimes \Gamma)(z)$ appartient à $\mathfrak{N}_{\psi \otimes \varphi \otimes \omega_{\hat{\Delta}^{1 / 4}} \dot{\xi}}$ De même, grâce au corollaire I.4 et au lemme I.12, on a:

$$
\begin{aligned}
\left(\psi \otimes \varphi \otimes \omega_{\hat{\Delta}^{-1 / 4}} \circ \kappa\right)((i \otimes \Gamma)(z)) & =\left(\psi \otimes \varphi \otimes \omega_{\hat{\Delta}^{1 / 4} \hat{\jmath} \xi}\right)((i \otimes \Gamma)(z)) \\
& =(\psi \otimes \varphi)(z)\left\|\hat{\Delta}^{-1 / 4} \hat{J} \xi\right\|^{2} \\
& =(\psi \otimes \varphi)(z)\left\|\hat{\Delta}^{1 / 4} \xi\right\|^{2}
\end{aligned}
$$

et $(i \otimes \Gamma)(z)$ appartient à $\mathfrak{R}_{\psi \otimes \varphi \otimes_{\hat{\Delta}^{-1 / 4}}} \propto \kappa$. Ces deux expressions de l'énoncé ont donc un sens.

De plus d'après [5], 4.2.1., (P2) et $(\mathrm{P} 4)$, on a:

$$
\psi \otimes \varphi \otimes \omega_{\hat{\Delta}^{1 / 4 \xi}}=\left(\psi \otimes \varphi \circ \kappa \otimes \omega_{\xi}\right)\left(\left(1 \otimes \hat{\Delta} \otimes \hat{\Delta}^{1 / 2}\right) .\right)
$$

et, d'après le lemme I.12:

$$
\psi \otimes \varphi \otimes \omega_{\hat{\Delta}^{-1 / 4}} \circ \kappa=\left(\psi \otimes \varphi \circ \kappa \otimes \omega_{\xi} \circ \kappa\right)\left(\left(1 \otimes \hat{\Delta} \otimes \hat{\Delta}^{1 / 2}\right) .\right)
$$

Par ailleurs, grâce à I.2. (ii), on a :

$$
\begin{gathered}
(i \otimes \Gamma)\left(1 \otimes\left(\hat{\Delta}^{1 / 2}\right)_{\varepsilon}\right)\left(1 \otimes\left(\hat{\Delta}^{1 / 2}\right)_{\varepsilon} \otimes 1\right) \\
\left.=\left(1 \otimes \hat{\Delta}^{1 / 2} \otimes \hat{\Delta}^{1 / 2}\right)_{\varepsilon}\right)\left(1 \otimes\left(\hat{\Delta}^{1 / 2}\right)_{\varepsilon} \otimes 1\right) \\
\nearrow 1 \otimes \hat{\Delta} \otimes \hat{\Delta}^{1 / 2} .
\end{gathered}
$$

Donc, grâce à (P3), on trouve: 


$$
\begin{aligned}
& \left(\psi \otimes \varphi \otimes \omega_{\hat{\Delta}^{1 / 4} \xi}\right)\left((i \otimes \Gamma)\left(y^{*}\right)(x \otimes 1)\right)= \\
& \quad=\lim _{\varepsilon \rightarrow 0}\left(\psi \otimes \varphi \circ \kappa \otimes \omega_{\xi}\right)\left((i \otimes \Gamma)\left(1 \otimes\left(\hat{\Delta}^{1 / 2}\right)_{\varepsilon}\right)\left(1 \otimes\left(\hat{\Delta}^{1 / 2}\right)_{\varepsilon} \otimes 1\right)(i \otimes \Gamma)\left(y^{*}\right)(x \otimes 1)\right) \\
& \quad=\lim _{\varepsilon \rightarrow 0}\left(\psi \otimes \varphi \circ \kappa \otimes \omega_{\xi}\right)\left((i \otimes \Gamma)\left(\left(1 \otimes \hat{\Delta}^{1 / 2}\right)_{\varepsilon}\right) y^{*}\right)\left(\left(1 \otimes\left(\hat{\Delta}^{1 / 2}\right)_{\varepsilon}\right) x \otimes 1\right) \\
& \left.\quad=\lim _{\varepsilon \rightarrow 0}\left(\psi \otimes \varphi \circ \kappa \otimes \omega_{\xi} \circ \kappa\right)\left(\left(1 \otimes \hat{\Delta}^{1 / 2}\right)_{\varepsilon}\right) y^{*} \otimes 1\right)(i \otimes \Gamma)\left(\left(1 \otimes\left(\hat{\Delta}^{1 / 2}\right)_{\varepsilon}\right) x\right)
\end{aligned}
$$

d'après le corollaire I.10, car $\left(1 \otimes\left(\hat{\Delta}^{1 / 2}\right)_{\varepsilon}\right) x$ et $\left(1 \otimes\left(\hat{\Delta}^{1 / 2}\right)_{\varepsilon}\right) y$ appartiennent à $\mathfrak{N}_{\psi \otimes \varphi \circ \kappa}$.

$$
\begin{aligned}
& =\lim _{\varepsilon \rightarrow 0}\left(\psi \otimes \varphi \circ \kappa \otimes \omega_{\xi} \circ \kappa\right)\left((i \otimes \Gamma)\left(1 \otimes\left(\hat{\Delta}^{1 / 2}\right)_{\varepsilon}\right)\left(1 \otimes\left(\hat{\Delta}^{1 / 2}\right)_{\varepsilon} \otimes 1\right)\left(y^{*} \otimes 1\right)(i \otimes \Gamma)(x)\right. \\
& =\left(\psi \otimes \varphi \otimes \omega_{\hat{\Delta}^{-1 / 4 \xi} \circ \kappa}\right)\left(\left(y^{*} \otimes 1\right)(i \otimes \Gamma)(x)\right)
\end{aligned}
$$

ce qui provient des résultats énoncés ci-dessus.

Corollaire 1.14: Soient $x$ et $y$ dans $\mathfrak{R}_{\varphi}, \xi$ dans $\mathscr{D}\left(\hat{\Delta}^{1 / 4}\right) \cap \mathscr{D}\left(\hat{\Delta}^{-1 / 4}\right)$. On $a$ :

$$
\left(\varphi \otimes \omega_{\Delta^{1 / 4 \xi}}\right)\left(\Gamma\left(y^{*}\right)(x \otimes 1)\right)=\left(\varphi \otimes \omega_{\Delta^{-1 / 4} \xi \circ \kappa}\right)\left(\left(y^{*} \otimes 1\right) \Gamma(x)\right) .
$$

Démonstration: Il suffit d'appliquer le corollaire précédent à un poids $\psi$ fini et à des éléments de la forme $1 \otimes x$ et $1 \otimes y$.

Lemme 1.15: Soit $x$ dans $A \otimes \mathcal{L}(H)$. Les deux propositions suivantes sont équivalentes:

(i) $x \in A \otimes M^{\prime}$

(ii) $(x \otimes 1)(1 \otimes W)=(1 \otimes W)(x \otimes 1)$.

Démonstration: Le fait que (i) entraîne (ii), résulte de [5], 2.1.1.. Réciproquement, supposons (ii). Soit $a$ dans $M$. On a:

$$
\begin{aligned}
(x \otimes 1)(1 \otimes \Gamma(a)) & =(x \otimes 1)(1 \otimes W)(1 \otimes 1 \otimes a)\left(1 \otimes W^{*}\right) \quad \text { d'après [5], } 2.2 .5(\mathrm{a}) \\
& =(1 \otimes W)(x \otimes a)\left(1 \otimes W^{*}\right) \quad \text { par hypothèse } \\
& =(1 \otimes W)(1 \otimes 1 \otimes a)\left(1 \otimes W^{*}\right)(x \otimes 1) \quad \text { par hypohèse } \\
& =(1 \otimes \Gamma(a))(x \otimes 1)
\end{aligned}
$$

donc, $(x \otimes 1)$ commute à $C \otimes \Gamma(M)$, comme il commute à $\mathbb{C} \otimes C \otimes M$, il commute à $\mathbb{C} \otimes(\Gamma(M) \cup \boldsymbol{C} \otimes M)^{\prime \prime}=\mathbb{C} \otimes M \otimes M$ (d'après [4], III.4 appliqué à $\mathbb{K}^{s}$ ), d'où (i).

Lemme 1.16: Soient $x$ dans $\mathfrak{N}_{\varphi}, \quad y$ dans $\mathfrak{N}_{\varphi} \cap \mathfrak{\Re}_{\varphi_{\circ} \kappa} \quad$ L'élément $\Lambda_{\varphi \otimes \varphi}\left(\Gamma(y)(x \otimes 1)\right.$ apartient à $\mathscr{D}\left((\hat{\Delta} \otimes \hat{\Delta})^{-1 / 2}\right)$.

Démonstration: On a: 


$$
\begin{aligned}
& \left((\hat{\Delta} \otimes \hat{\Delta})^{-1 / 2}\right)_{\mathrm{\varepsilon}} \Lambda_{\varphi \otimes \varphi}(\Gamma(y)(x \otimes 1))= \\
& =\left((\Delta \otimes \Delta)^{-1 / 2}\right)_{\varepsilon} W\left(\Lambda_{\varphi}(x) \otimes \Lambda_{\varphi}(y)\right) \text { d'après [5], 2.2.1. } \\
& =W\left(1 \otimes\left(\hat{\Delta}^{-1 / 2}\right)_{\varepsilon}\right)\left(\Lambda_{\varphi}(x) \otimes \Lambda_{\varphi}(y)\right) \quad \text { d'aprés I.2.(ii) et [5], 2.2.5. (a) } \\
& =W\left(\Lambda_{\varphi}(x) \otimes\left(\hat{\Delta}^{-1 / 2}\right)_{\varepsilon} \Lambda_{\varphi}(y)\right) \text {. }
\end{aligned}
$$

Il en résulte que:

$$
\begin{aligned}
\|\left(\left(\hat{\Delta} \otimes \hat{\Delta}^{-1 / 2}\right)_{\varepsilon} \Lambda_{\varphi \otimes \varphi}(\Gamma(y)(x \otimes 1)) \|^{2}\right. & =\left\|\Lambda_{\varphi}(x) \otimes\left(\hat{\Delta}^{-1 / 2}\right)_{\varepsilon} \Lambda_{\varphi}(y)\right\|^{2} \\
& =\varphi\left(x^{*} x\right) \varphi\left(\left(\hat{\Delta}^{-1 / 2}\right)_{\varepsilon} y^{*} y\right) \\
& \underset{\varepsilon \rightarrow 0}{\longrightarrow} \varphi\left(x^{*} x\right) \varphi \circ \kappa\left(y^{*} y\right)<\infty
\end{aligned}
$$

d'après [5], 4.2.1., d'où le résultat.

\section{Préliminaires concernant les produits croisés}

On conserve dans ce chapitre les notations du chapitre précédent. De plus, $A$ sera supposée réalisée dans un espace hilbertien $K$. D'autre part, $\alpha$ désignera une action à droite de $\mathbb{K}$ sur $A$, au sens de [4], I.1.

On utilisera la terminologie et les notations de [4]. Toutefois, nous n'utiliserons que des actions à droite, que nous appellerons simplement "action." (Rappelons qu'une action à gauche de $\mathbb{K}$ est une action à droite de $\mathbb{K}^{s}$ ).

Enfin, $\alpha_{i}$ désignera une action de l'algèbre de $\mathrm{Kac} \mathbb{K}_{i}$ sur l'algèbre de von Neumann $A_{i}$, réalisée dans l'espace hilbertien $K_{i}(i=1,2)$.

Définition 11.1: On pose: $A^{\alpha}=\{x \in A: \alpha(x)=x \otimes 1\}$. On dira que $A^{\alpha}$ est la sous-algèbre des éléments invariants par $\alpha$.

Proposition 11.2: L'application $T_{a}=(i \otimes \varphi) \alpha$ est un poids opératoriel normal fidèle de $A$ sur $A^{\alpha}$.

Démonstration: Soit $x$ dans $A^{+}$. Il est clair que $T_{\infty} x$ appartient à $\operatorname{ext}^{+} A$; en notant encore $\alpha$ le prolongement canonique de $\alpha$ à $\operatorname{ext}^{+} A$, on a:

$$
\begin{aligned}
\alpha\left(T_{a x} x\right) & =\alpha(i \otimes \varphi) \alpha(x) \\
& =(i \otimes i \otimes \varphi)(\alpha \otimes i) \alpha(x) \\
& =(i \otimes i \otimes \varphi)(i \otimes \Gamma) \alpha(x) \quad \text { d'après [4], I.1. } \\
& =(i \otimes \varphi) \alpha(x) \otimes 1 \quad \text { d'après [17], Th. III.34 (N Kiii) } \\
& =T_{\alpha} x \otimes 1
\end{aligned}
$$

donc $T_{\infty} x$ appartient à $\operatorname{ext}^{+} A^{\infty}$. 
Comme $i \otimes \varphi$ set un poids opératoriel normal fidèle de $A \otimes M$ sur $A$, la seule vérification non triviale qui reste à effectuer est: soit a dans $A^{\infty}$, on a:

$$
\begin{aligned}
T_{a(}\left(a^{*} x a\right) & =(i \otimes \varphi)\left(\left(a^{*} \otimes 1\right) \alpha(x)(a \otimes 1)\right) \\
& =a^{*}(i \otimes \varphi) \alpha(x) a \\
& =a^{*} T_{a}(x) a
\end{aligned}
$$

ce qui achève la démonstration.

Définition II.3.: On dira que l'action $\alpha$ est intégrable si le poids opératoriel $T_{a}$ drfini en II.2. est semi-fini.

Remarquons que cette notion est stable par équivalence forte ([4], I.10), mais pas par équivalence.

Dans le cas où $\beta$ désigne une action d'un groupe localement compact $G$ sur $A$, l'action $\beta^{\alpha}$ de $K A(G)$ ([5], 8.1.1., [4], I.3.) sur $A$ sera intégrable si et seulement si l'ensemble $\left\{x \in A^{+} ; \int_{G} \beta_{s}(x) d s \in A^{+}\right\}$est faiblement dense dans $A^{+}$.

Proposition 11.4: (i) L'action $\Gamma$ ([4], exemple suivant I.1.) est intégrable (ii) L'action duale $\tilde{\alpha}$ ([4], II.7) est intégrable.

Démonstration: (i) Cela résulte trivialement de [17], Th.III.34 (NKiii) (ii) Soit a dans $\hat{M}^{\prime}$. On a:

$$
\begin{aligned}
T_{\tilde{\alpha}}\left(1 \otimes a^{*} a\right) & =\left(i \otimes \hat{\varphi}^{\prime}\right) \tilde{\alpha}\left(1 \otimes a^{*} a\right) \\
& =\left(i \otimes \hat{\varphi}^{\prime}\right)\left(1 \otimes \hat{\Gamma}^{\prime}\left(a^{*} a\right)\right) \quad \text { d'après [4], II. } 56 . \\
& =\hat{\varphi}^{\prime}\left(a^{*} a\right) \cdot 1 \quad \text { d'après [17] III. } 34 \text { appliqué à } \boldsymbol{K}^{\wedge^{\prime}}
\end{aligned}
$$

Donc, si a appartient à $\mathfrak{N}_{\tilde{\varphi}}, 1 \otimes a$ appartient à $\mathfrak{N}_{T \tilde{\alpha}}$, qui est ainsi dense dans $\mathscr{W}^{*}(\alpha)$.

Définition II.5: On dira que $\alpha$ vérifie la propriété (B) si

$$
\alpha(A)=\mathscr{W}^{*}(a)^{\tilde{\alpha}}
$$

(L'inclusion $\alpha(A) \subset \mathscr{W}^{*}(\alpha)^{\tilde{\alpha}}$ est évidente, cf [4], V. (ii)).

Proposition II.6: (i) On $a$ : $\mathscr{W}^{*}(\alpha)^{\widetilde{\alpha}}=\mathscr{W}^{*}(\alpha) \cap \mathcal{L}(K) \otimes M$

$$
=\mathscr{W}^{*}(\alpha) \cap A \otimes M
$$

(ii) La propriété (B) est stable par équivalence (cf. [4], I.10).

Démonstration: (i) Ii réulte de [4], II.6(i) que: 
$\mathscr{W}^{*}(\alpha)^{\tilde{\alpha}}=\{x \in \mathscr{W} *(\alpha) ;(x \otimes 1)$ commute à $1 \otimes(J \hat{J} \otimes J \hat{J}) \mathscr{W} *(J \hat{J} \otimes J \hat{J})\}$ $=\mathscr{W} *(\alpha) \cap \mathcal{L}(K) \otimes M, \quad$ d'après le lemme I.15 appliqué à $\mathbb{K}^{\mathrm{s}}$

(cf [17], VII. 10.).

Comme $\mathscr{W W}^{*}(\alpha) \subset A \otimes \mathcal{L}(H)$, la deuxième égalité est évidente.

(ii) Soient $\alpha_{1}, \alpha_{2}$ deux actions telles que $\alpha_{1} \sim \alpha_{2}(\Phi, \Psi, U)$. Il résulte de [4], II.3. que:

$$
\begin{aligned}
U \mathscr{W} \mathcal{W}^{*}\left(\alpha_{1}\right) U^{*} & =\left(\Phi^{-1} \otimes \bar{\Psi}^{-1}\right)\left(\mathscr{W} *\left(\alpha_{2}\right)\right) \\
& \subset\left(\Phi^{-1} \otimes \bar{\Psi}^{-1}\right)\left(A_{2} \otimes \mathcal{L}\left(H_{2}\right)\right)=A_{1} \otimes \mathcal{L}\left(H_{1}\right) .
\end{aligned}
$$

Or, d'après (i), on a:

$$
\begin{aligned}
U \mathscr{W} *\left(\alpha_{1}\right)^{\tilde{\omega}_{1} U^{*}} & =U \mathscr{W} *\left(\alpha_{1}\right) U^{*} \cap \mathcal{L}(K) \otimes M_{1}, \text { car } U \in \mathcal{L}(K) \otimes M_{1} \\
& =U \mathscr{W} *\left(\alpha_{1}\right) U^{*} \cap A_{1} \otimes M_{1}, \quad \text { d'après ce qui précède. }
\end{aligned}
$$

D'où:

$$
\begin{aligned}
(\Phi \otimes \bar{\Psi})\left(U \mathscr{W} *\left(\alpha_{1}\right)^{\tilde{\omega}_{1}} U^{*}\right) & =\mathscr{W} *\left(\alpha_{2}\right) \cap A_{2} \otimes M_{2} \\
& =\mathscr{W}^{*}\left(\alpha_{2}\right)^{\tilde{\omega}_{1}} \quad \text { d'après (i). }
\end{aligned}
$$

Supposons que $\alpha_{i}$ vérifie (B). On a:

$\mathscr{W W}^{*}\left(\alpha_{2}\right)^{\tilde{\alpha}_{2}}=(\Phi \otimes \bar{\Psi})\left(U \alpha_{1}\left(A_{1}\right) U^{*}\right)=\alpha_{2}\left(A_{2}\right)$, d'après [4], II.3, d'où (ii).

Proposition II.7: Soit $U$ un $1_{K}^{\complement(K)}$-cocycle. On a:

(i) $U^{*} r$ est un $1_{\boldsymbol{K}^{\sigma}}^{\mathcal{L}(K)}$-cocycle.

(ii) Si U implémente une action $\alpha$ de $\mathbb{K}$ sur $A, U^{*}$ implémente une action de $\boldsymbol{K}^{\sigma}$ sur $A^{\prime}$, que l'on notera $\alpha^{\prime}$.

Démonstration: Il résulte de [4], I.6. que:

$$
(i \otimes \Gamma)\left(U^{*}\right)=(1 \otimes \sigma)\left(U^{*} \otimes 1\right)(1 \otimes \sigma)\left(U^{*} \otimes 1\right)
$$

donc $(i \otimes \varsigma \Gamma)\left(U^{*}\right)=\left(U^{*} \otimes 1\right)(1 \otimes \sigma)\left(U^{*} \otimes 1\right)(1 \otimes \sigma)$, d'où (i).

Si l'on suppose $U\left(A \otimes C_{H}\right) U^{*} \subset A \otimes \mathcal{L}(H)$, on a successivement:

$$
\begin{aligned}
& U\left(A^{\prime} \otimes \mathcal{L}(H)\right) U^{*} \supset A^{\prime} \otimes C_{H} \\
& U^{*}\left(A^{\prime} \otimes C_{H}\right) U \subset A^{\prime} \otimes \mathcal{L}(H), \quad \text { d'où (ii). }
\end{aligned}
$$

Définition II.8: (i) On appellera algèbre saturée par $\alpha$ l'ensemble

Sat $\alpha=\{x \in A \otimes M ;(\alpha \otimes i)(x)=(i \otimes \Gamma)(x)\}$ 
(ii) On dira que 1'action $\alpha$ est saturée si $\alpha(A)=$ Sat $\alpha$.

Remarquons qu'on a toujours $\alpha(A) \subset$ Sat $\alpha$. Cette notion a été introduite en [18].

Lemme II.9: Soit $U$ un $1_{K}^{\mathcal{L}(K)}$-cocycle. On a:

(i) $U^{*}\left(C_{K} \otimes \hat{M}^{\prime}\right) U \subset \mathcal{L}(K) \otimes \hat{M}^{\prime}$

(ii) Si l'on suppose que $U$ implémente une action $\alpha$ de $\boldsymbol{K}$ sur $A$, on a

$$
\begin{aligned}
& \mathscr{W}^{*}(\alpha) \subset U\left(\mathcal{L}(K) \otimes \hat{M}^{\prime}\right) U^{*} \\
& \mathscr{W}^{*}(\alpha)^{\tilde{\alpha}} \subset U\left(\mathcal{L}(K) \otimes C_{H}\right) U^{*} \cap A \otimes M .
\end{aligned}
$$

Démonstration: En appliquant [17], I. 2. à $\boldsymbol{K}^{\wedge^{\prime}}$, il vient d'après [17], VII. 10. :

$$
\left(\{\sigma(\hat{J} \otimes \hat{J}) W(\hat{J} \otimes \hat{J}) \sigma\} \cup C_{K} \otimes \mathcal{L}(H)\right)^{\prime \prime}=\hat{M}^{\prime} \otimes \mathcal{L}(H) .
$$

On en déduit que:

$$
\begin{aligned}
\left(U^{*}\right. & \otimes 1)\left(C_{K} \otimes \hat{M}^{\prime} \otimes \mathcal{L}(H)\right)(U \otimes 1)= \\
& =\left(U^{*} \otimes 1\right)\left(\{1 \otimes \sigma(\hat{J} \otimes \hat{J}) W(\hat{J} \otimes \hat{J}) \sigma\} \cup C_{K} \otimes C_{H} \otimes \mathcal{L}(H)\right)^{\prime \prime}(U \otimes 1) \\
& =\left(\left\{\left(U^{*} \otimes 1\right)(1 \otimes \sigma(\hat{J} \otimes \hat{J} J) W(\hat{J} \otimes \hat{J}) \sigma)(U \otimes 1)\right\} \cup C_{K} \otimes C_{H} \otimes \mathcal{L}(H)\right)^{\prime \prime}
\end{aligned}
$$

Or, d'après [4], I.5. (ii) on a:

$(1 \otimes \sigma(\hat{J} \otimes \hat{J}) W(\hat{J} \otimes \hat{J}) \sigma)(U \otimes 1)\left(1 \otimes \sigma(\hat{J} \otimes \hat{J}) W^{*}(\hat{J} \otimes \hat{J}) \sigma\right)$

$=(i \otimes \Gamma)(U)$

$=(U \otimes 1)(1 \otimes \sigma)(U \otimes 1)(1 \otimes \sigma)$ d'après [4], I.6.

Donc,

$\left(U^{*} \otimes 1\right)(1 \otimes \sigma(\hat{J} \otimes \hat{J}) W(\hat{J} \otimes \hat{J}) \sigma)(U \otimes 1)$

$=(1 \otimes \sigma)(U \otimes 1)(1 \otimes \sigma)(1 \otimes \sigma(\hat{J} \otimes \hat{J}) W(\hat{J} \otimes \hat{J}) \sigma)$

$\in\left(\mathcal{L}(K) \otimes \boldsymbol{C}_{H} \otimes M \cup \boldsymbol{C}_{K} \otimes \hat{M}^{\prime} \otimes M\right)^{\prime \prime} \quad$ d'après [5], 2.1.1 et 3.1.5.

$=\mathcal{L}(K) \otimes \hat{M}^{\prime} \otimes M$.

Il en résulte que:

$\left(U^{*} \otimes 1\right)\left(C_{K} \otimes \hat{M}^{\prime} \otimes \mathcal{L}(H)\right)(U \otimes 1) \subset\left(\mathcal{L}(K) \otimes \hat{M}^{\prime} \otimes M \cup C_{K} \otimes C_{M} \otimes \mathcal{L}(H)\right)^{\prime \prime}$

$$
=\mathcal{L}(K) \otimes \hat{M}^{\prime} \otimes \mathcal{L}(H) \quad \text { d'où (i). }
$$

On a:

$$
\begin{aligned}
\mathscr{W}^{*}(\alpha) & =\left(\alpha(A) \cup C_{K} \otimes \hat{M}^{\prime}\right)^{\prime \prime} \\
& =\left(U\left(A \otimes C_{H}\right) U^{*} \cup C_{K} \otimes \hat{M}^{\prime}\right)^{\prime \prime}
\end{aligned}
$$


$=U\left(A \otimes C_{H} \cup U^{*}\left(\boldsymbol{C}_{K} \otimes \hat{M}^{\prime}\right) U\right)^{\prime \prime} U^{*}$

$\subset U\left(A \otimes \mathcal{C}_{H} \cup \mathcal{L}(K) \otimes \hat{M}^{\prime}\right)^{\prime \prime} U^{*}$ d'parès (i)

$=U\left(\mathcal{L}(K) \otimes \hat{M}^{\prime}\right) U^{*}$, d'où la première partie de (ii).

On a alors:

$$
\begin{aligned}
\mathscr{W}^{*}(\alpha)^{\tilde{\alpha}} & =\mathscr{W} *(\alpha) \cap \mathcal{L}(K) \otimes M \quad \text { d'après II.6. (i) } \\
& \subset U\left(\mathcal{L}(K) \otimes \hat{M}^{\prime}\right) U^{*} \cap \mathcal{L}(K) \otimes M \quad \text { d'après ce qui précède } \\
& =U\left(\mathcal{L}(K) \otimes \hat{M}^{\prime} \cap \mathcal{L}(K) \otimes M\right) U^{*} \quad \text { car } U \in \mathcal{L}(K) \otimes M \\
& =U\left(\mathcal{L}(K) \otimes \mathcal{C}_{H}\right) U^{*} \quad \text { d'après [17]. I.4. }
\end{aligned}
$$

d'où (ii)

Lemme II.10: Soit $U$ un $1_{K}^{\mathcal{L}(K)}$-cocycle qui implémente $\alpha$. Pour tout $x$ de $A \otimes \mathcal{L}(H)$, on a

$$
(\alpha \otimes i)(x)=(U \otimes 1)(1 \otimes \sigma)(x \otimes 1)(1 \otimes \sigma)\left(U^{*} \otimes 1\right)
$$

Démonstration: Soient $x_{1}$ dans $A$ et $x_{2}$ dens $\mathcal{L}(H)$. On a:

$$
\begin{aligned}
(\alpha \otimes i)\left(x_{1} \otimes x_{2}\right) & =\alpha\left(x_{1}\right) \otimes x_{2} \\
& =U\left(x_{1} \otimes 1\right) U^{*} \otimes x_{2} \\
& =(U \otimes 1)\left(x_{1} \otimes 1 \otimes x_{2}\right)\left(U^{*} \otimes 1\right) \\
& =(U \otimes 1)(1 \otimes \sigma)\left(x_{1} \otimes x_{2} \otimes 1\right)(1 \otimes \sigma)\left(U^{*} \otimes 1\right)
\end{aligned}
$$

d'où le résultat, par linéarité et continuité.

Lemme II.11: Soit $U$ un $1_{K}^{\mathcal{L}(K)}$-cocycle qui implémente $\alpha$. On a:

$$
\begin{aligned}
& A \otimes \mathcal{L}(H) \cap U\left(\mathcal{L}(K) \otimes \hat{M}^{\prime}\right) U^{*} \\
& \quad=\left\{x \in A \otimes \mathcal{L}(H) ;(\alpha \otimes i)(x)=(1 \otimes W)(1 \otimes \sigma)(x \otimes 1)(1 \otimes \sigma)\left(1 \otimes W^{*}\right)\right\} .
\end{aligned}
$$

Démonstration: Il résulte du fait que $U$ est un cocycle et de [4], I.5.3 que

$(1 \otimes W)(1 \otimes \sigma)(U \otimes 1)(1 \otimes \sigma)\left(1 \otimes W^{*}\right)=(U \otimes 1)(1 \otimes \sigma)(U \otimes 1)(1 \otimes \sigma)$ $\mathrm{d}^{\prime}$ où

$(U \otimes 1)(1 \otimes \sigma)\left(1 \otimes W^{*}\right)(1 \otimes \sigma)\left(U^{*} \otimes 1\right)=(1 \otimes \sigma)\left(1 \otimes W^{*}\right)(U \otimes 1)(1 \otimes \sigma)$.

Il résulte alors de I.15. appliqué à $\mathbb{K}^{\wedge}$ qu'un élément $x$ de $A \otimes \mathcal{L}(H)$ appartient à $U\left(\mathcal{L}(K) \otimes \hat{M}^{\prime}\right) U^{*}$ si et seulement si $x \otimes 1$ commute à 
$(1 \otimes \sigma)\left(1 \otimes W^{*}\right)(U \otimes 1)(1 \otimes \sigma), \quad$ ou encore si $1^{\prime}$ on a

$(U \otimes 1)(1 \otimes \sigma)(x \otimes 1)(1 \otimes \sigma)\left(U^{*} \otimes 1\right)=(1 \otimes W)(1 \otimes \sigma)(x \otimes 1)(1 \otimes \sigma)\left(1 \otimes W^{*}\right)$

et le lemme résulte alors de II.10.

Proposition II.12: Soit $U$ un $1_{K}^{\mathcal{L}(K)}$-cocycle qui implémente $\alpha$. On a

$$
\begin{aligned}
\text { Sat } \alpha & =A \otimes \mathcal{L}(H) \cap U\left(\mathcal{L}(K) \otimes C_{H}\right) U^{*} \\
& =A \otimes M \cap U\left(\mathcal{L}(K) \otimes C_{H}\right) U^{*} .
\end{aligned}
$$

Démonstration: En utilisant [4], I.5. (iii), le lemme précédent fournit:

$$
\text { Sat } \begin{aligned}
\alpha & =A \otimes M \cap U\left(\mathcal{L}(K) \otimes \hat{M}^{\prime}\right) U^{*} \\
& =U\left(U^{*}(A \otimes M) U \cap \mathcal{L}(K) \otimes \hat{M}^{\prime}\right) U^{*} \\
& \subset U\left(\mathcal{L}(K) \otimes M \cap \mathcal{L}(K) \otimes \hat{M}^{\prime}\right) U^{*} \quad \text { car } U \in \mathcal{L}(K) \otimes M \\
& =U\left(\mathcal{L}(K) \otimes C_{H}\right) U^{*} \quad \text { d'après [17], I.4. }
\end{aligned}
$$

d'où la deuxième égalité. La premiére provient de ce que

$$
U\left(\mathcal{L}(K) \otimes C_{H}\right) U^{*} \subset \mathcal{L}(K) \otimes M
$$

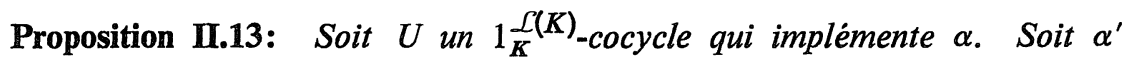
l'action de $\boldsymbol{K}^{\sigma}$ sur $A^{\prime}$ définie en II.7. (ii). Les deux propriétés suivantes sont équivalentes:

(i) $\alpha$ est saturée

(ii) $\alpha^{\prime}$ vérifie la condition $(A)$ de [4], III.2.

De plus, si (i) ou (ii) est vérifiée, $\alpha$ vérifie la condition $(B)$ de II.5.

Démonstration: Il résulte de II.12 que (i) équivaut à:

$$
U\left(A \otimes C_{H}\right) U^{*}=A \otimes \mathcal{L}(H) \cap U\left(\mathcal{L}(K) \otimes C_{H}\right) U^{*}
$$

et donc, successivement à:

$$
\begin{gathered}
U\left(A^{\prime} \otimes \mathcal{L}(H)\right) U^{*}=\left(A^{\prime} \otimes C_{H} \cup U\left(C_{K} \otimes \mathcal{L}(H) U^{*}\right)^{\prime \prime}\right. \\
\begin{aligned}
A^{\prime} \otimes \mathcal{L}(H) & =\left(U^{*}\left(A^{\prime} \otimes C_{H}\right) U \cup C_{K} \otimes \mathcal{L}(H)\right)^{\prime \prime} \\
& =\left(\alpha^{\prime}\left(A^{\prime}\right) \cup C_{K} \otimes \mathcal{L}(H)\right)^{\prime \prime}
\end{aligned}
\end{gathered}
$$

ce qui est l'énoncé de (ii).

D'autre part, il résulte de II.9.(ii) et II.12. que

$$
\mathscr{W}^{*}(\alpha)^{\tilde{\alpha}} \subset \text { Sat } \alpha \text {. }
$$

Donc, si $\alpha$ est saturée, on a $\mathscr{W}^{*}(\alpha)^{\tilde{\alpha}} \subset \alpha(A)$, ce qui implique $(B)$. 
Lemme II.14: On a:

$$
\mathscr{W} *(\alpha) \otimes M=\left((\alpha \otimes i) \alpha(A) \cup C_{K} \otimes \hat{M}^{\prime} \otimes M\right)^{\prime \prime} .
$$

Démonstration: On a:

$$
(\alpha \otimes i) \alpha(A) \subset \alpha(A) \otimes M
$$

donc,

$$
\begin{aligned}
\left((\alpha \otimes i) \alpha(A) \cup C_{K} \otimes \hat{M}^{\prime} \otimes M\right)^{\prime \prime} & \subset\left(\alpha(A) \otimes M \cup C_{K} \otimes \hat{M}^{\prime} \otimes M\right)^{\prime \prime} \\
& =\mathscr{W}^{*}(\alpha) \otimes M .
\end{aligned}
$$

Réciproquement,

$$
\mathscr{W}^{*}(\alpha) \otimes M=\left(\alpha(A) \otimes C_{H} \cup C_{K} \otimes \hat{M} \otimes M\right)^{\prime \prime} .
$$

Or, pour tout $x$ de $A$, on a:

$$
\begin{aligned}
(\alpha \otimes i) \alpha(x) & =(i \otimes \Gamma) \alpha(x) \\
& =(1 \otimes \sigma(\hat{J} \otimes \hat{J}) W(\hat{J} \otimes \hat{J}) \sigma)(\alpha(x) \otimes 1)\left(1 \otimes \sigma(\hat{J} \otimes \hat{J}) W^{*}(\hat{J} \otimes \hat{J}) \sigma\right)
\end{aligned}
$$

d'après [4], II.5. (ii)

donc:

$$
\alpha(A) \otimes C_{H} \subset\left(1 \otimes \sigma(\hat{J} \otimes \hat{J}) W^{*}(\hat{J} \otimes \hat{J}) \sigma\right)(\alpha \otimes i) \alpha(A)(1 \otimes \sigma(\hat{J} \otimes \hat{J}) W(\hat{J} \otimes \hat{J}) \sigma)
$$
d'où :

$\alpha(A) \otimes C_{H} \subset\left((\alpha \otimes i) \alpha(A) \cup C_{K} \otimes \hat{M}^{\prime} \otimes M\right)^{\prime \prime} \quad$ d'après [5], 2.1.1 et 3.1.5.

Dans ces conditions:

$$
\mathscr{W}^{*}(\alpha) \otimes M \subset\left((\alpha \otimes i) \alpha(A) \cup \boldsymbol{C}_{K} \otimes M^{\prime} \otimes M\right)^{\prime \prime}
$$

ce qui achève la démonstration.

Lemme II.15: (i) La restriction de $(i \otimes \varsigma)(\alpha \otimes i)$ à l'algèbre $B=$ $\left(\alpha(A) \cup C_{K} \otimes \mathcal{L}(H)\right)^{\prime \prime}$ est une action de $\mathbb{K}$ sur $B$. On la notera $r$.

(ii) $1 \otimes \sigma W^{*} \sigma$ est un $r$-cocycle

(iii) $\quad \alpha^{\sim \sim} \sim \gamma\left(\Theta(\alpha \otimes i), v, 1 \otimes W^{*} \sigma\right)$

oú $\sigma$ a été défini en [4], III.1; $v$ désigne l'isomorphisme canonique de $\mathbb{K}$ sur $\mathbb{K}^{\prime s}$ défini en [17], VII.8.).

(Ce résultat généralise [4], III.6 et III.8.)

Démonstration: On a: 


$$
\begin{aligned}
(i \otimes \varsigma)(\alpha \otimes i)(B) & =(i \otimes \varsigma)\left((\alpha \otimes i) \alpha(A) \cup C_{K} \otimes \mathcal{C}_{H} \otimes \mathcal{L}(H)\right)^{\prime \prime} \\
& =\left((i \otimes \varsigma)(\alpha \otimes i) \alpha(A) \cup C_{K} \otimes \mathcal{L}(H) \otimes C_{H}\right)^{\prime \prime}
\end{aligned}
$$

Or, pour tout $x$ dans $A$ :

$$
\begin{aligned}
(i \otimes \varsigma)(\alpha \otimes i .) \alpha(x) & =(i \otimes \varsigma)(i \otimes \Gamma) \alpha(x) \\
& =(i \otimes \varsigma \Gamma) \alpha(x) \\
& =(1 \otimes \sigma W \sigma)(\alpha(x) \otimes 1)\left(1 \otimes \sigma W^{*} \sigma\right) \quad \text { d'après [4], I.5. (i) }
\end{aligned}
$$

Donc:

$$
\begin{aligned}
(i \otimes \varsigma)(\alpha \otimes i) \alpha(A) & \subset\left(\alpha(A) \otimes C_{H} \cup \boldsymbol{C}_{K} \otimes \mathcal{L}(H \otimes H)\right)^{\prime \prime} \\
& =B \otimes \mathcal{L}(H) .
\end{aligned}
$$

Il résulte alors de $(*)$ que:

$$
\begin{aligned}
(i \otimes \varsigma)(\alpha \otimes i)(B) & \subset\left(B \otimes \mathcal{L}(H) \cup C_{K} \otimes \mathcal{L}(H) \otimes C_{H}\right)^{\prime \prime} \\
& =B \otimes \mathcal{L}(H) .
\end{aligned}
$$

Comme $(i \otimes \varsigma)(\alpha \otimes i)$ est une action de $\mathbb{K}$ sur $A \otimes \mathcal{L}(H)$ ([4], III.6), il en résulte que sa restriction à $B$ est une action sur $B$, d'où (i). I1 résulte de [5], 2.1.1 que $1 \otimes \sigma W^{*} \sigma \in C_{K} \otimes \mathcal{L}(H) \otimes M \subset B \otimes M$. Par ailleurs, c'est un $(i \otimes \varsigma)$ $(\alpha \otimes i)$-cocycle ([4], III.6). On en déduit donc (ii).

L'application $\Theta(\alpha \otimes i)$ est un isomorphisme de $B$ sur $\mathscr{W} \mathcal{W}^{*}(\widetilde{\alpha})$.

Dans ces conditions, les démonstrations de [4], III. 7 et 8 , conduisent à (iii) en $y$ remplaçant $A \otimes \mathcal{L}(H)$ par $B$ (La propriété $(A)$ n'y intervenait en effet que pour montrer que $\Theta(\alpha \otimes i)$ est un isomorphisme de $A \otimes \mathcal{L}(H)$ sur $\mathscr{W} *(\widetilde{\alpha}))$.

Lemme 11.16: Soient $\alpha_{1}$ et $\alpha_{2}$ deux actions équivalentes telles que

$$
\alpha_{2} \sim \alpha_{1}(\Phi, \Psi, U)
$$

On a, pour tout $x$ de $A_{1}$ :

$$
\alpha_{1}(x)=U^{*}(x \otimes 1) U \Leftrightarrow \Phi(x) \in A_{2}^{\alpha} .
$$

Démonstration: Pour $x$ dans $A_{1}$, on a en effet

$$
\alpha_{2}(\Phi(x))=(\Phi \otimes \Psi)\left(U \alpha_{1}(x) U^{*}\right)
$$

Donc $\alpha_{1}(x)=U^{*}(x \otimes 1) U$ équivaut à $\alpha_{2}(\Phi(x))=(\Phi \otimes \Psi)(x \otimes 1)=\Phi(x) \otimes 1$ d'où le résultat.

Lemme 11.17: Pour toute algébre de von Neumann B, on a 
(i) $\beta=(i \otimes \varsigma)(\alpha \otimes i)$ est une action de $\mathbb{K}$ sur $A \otimes B$

(ii) $(A \otimes B)^{\beta}=A^{\infty} \otimes B$.

Démonstration: Il est clair que $\beta(A \otimes B) \subset A \otimes B \otimes M$. De plus:

$$
\begin{aligned}
(\beta \otimes i) \beta & =((i \otimes \varsigma)(\alpha \otimes i) \otimes i)(i \otimes \varsigma)(\alpha \otimes i) \\
& =(i \otimes \varsigma \otimes i)(\alpha \otimes i \otimes i)(i \otimes \varsigma)(\alpha \otimes i) \\
& =(i \otimes \varsigma \otimes i)(i \otimes i \otimes \varsigma)(\alpha \otimes i \otimes i)(\alpha \otimes i) \\
& =(i \otimes(\varsigma \otimes i)(i \otimes \varsigma))((i \otimes \Gamma) \alpha \otimes i) \\
& =(i \otimes(\varsigma \otimes i)(i \otimes \varsigma)(\Gamma \otimes i))(\alpha \otimes i) \\
& =(i \otimes(i \otimes \Gamma) \varsigma)(\alpha \otimes i) \quad \text { d'après I.5. } \\
& =(i \otimes i \otimes \Gamma)(i \otimes \varsigma)(\alpha \otimes i) \\
& =(i \otimes i \otimes \Gamma) \beta
\end{aligned}
$$

D'autre part, il est clair que $A^{\alpha} \otimes B \subset(A \otimes B)^{\beta}$. Réciproquement, soit $x$ dans $(A \otimes B)^{\beta}$. On a $\beta(x)=x \otimes 1$, c'est-à-dire:

$$
(\alpha \otimes i)(x)=(i \otimes \sigma)(x \otimes 1) .
$$

Pour tout $\Omega$ dans $B_{*}^{+}$, on a $(i \otimes \Omega)(x) \in A$, puis:

$$
\begin{aligned}
\alpha((i \otimes \Omega)(x)) & =(i \otimes i \otimes \Omega)(\alpha \otimes i)(x) \\
& =(i \otimes i \otimes \Omega)(i \otimes \varsigma)(x \otimes 1) \\
& =(i \otimes \Omega \otimes i)(x \otimes 1) \\
& =(i \otimes \Omega)(x) \otimes 1
\end{aligned}
$$

donc $(i \otimes \Omega)(x) \in A^{\alpha}$.

Pour tout $y$ de $\left(A^{o s}\right)^{\prime}$ on a donc

$$
\begin{aligned}
(i \otimes \Omega)(x(y \otimes 1)) & =(i \otimes \Omega)(x) y \\
& =y(i \otimes \Omega)(x) \\
& =(i \otimes \Omega)((y \otimes 1) x)
\end{aligned}
$$

d'où $x(y \otimes 1)=(y \otimes 1) x$, et on en déduit $x \in A^{\alpha} \otimes B$ d'où (ii).

III. Poids relativement invariants et formes canoniques

On conserve les notations du chapitre précédent.

Définition III.1: On dira qu'un poids $\psi$ de $P(A)$ est $\hat{\Delta}^{-1}$-relativement invariant par rapport à $\alpha$ s'il vérifie les conditions suivantes: 
(i) $(\psi \otimes i)(\alpha(x))=\psi(x) \hat{\Delta}^{-1} \quad\left(\forall x \in A^{+}\right)$

(ii) $\left(\psi \otimes \omega_{\hat{\Delta}^{1 / 4} \xi}\right)\left(\alpha\left(y^{*}\right)(x \otimes 1)\right)=\left(\psi \otimes \omega_{\hat{\Delta}^{-1 / 4}} \circ \kappa\right)\left(\left(y^{*} \otimes 1\right) \alpha(x)\right)$ $\left(\forall x, y \in \mathfrak{N}_{\psi}, \forall \xi \in \mathscr{D}\left(\hat{\Delta}^{1 / 4}\right) \cap \mathscr{D}\left(\hat{\Delta}^{-1 / 4}\right)\right)$.

Remarquons que (i) implique que pour $x$ et $y$ dans $\mathfrak{R}_{\psi}$ et $\xi$ dans $\mathscr{D}\left(\hat{\Delta}^{1 / 4}\right) \cap$ $\left(\hat{\Delta}^{-1 / 4}\right)$ on a:

$$
\left(\psi \otimes \omega_{\hat{\Delta}^{-1 / 4} \xi}\right)\left(\alpha\left(y^{*} y\right)\right)=\psi\left(y^{*} y\right)\left\|\hat{\Delta}^{-1 / 4} \xi\right\|^{2} .
$$

et

$$
\begin{aligned}
\left(\psi \otimes \omega_{\hat{\Delta}-1 / 4 \xi} \circ \kappa\right)\left(\alpha\left(x^{*} x\right)\right) & =\left(\psi \otimes \omega_{\hat{\Delta}^{1 / 4} \hat{\jmath} \xi}\right)\left(\alpha\left(x^{*} x\right)\right) \text { d'après I.12 } \\
& =\psi\left(x^{*} x\right)\left\|\hat{\Delta}^{-1 / 4} \hat{J} \xi\right\|^{2} \\
& =\psi\left(x^{*} x\right)\left\|\hat{\Delta}^{1 / 4} \xi\right\|^{2}
\end{aligned}
$$

les deux membres de l'égalité (ii) ont alors un sens.

(le cas échéant, on dira invariant à la place de 1-relativement invariant).

Proposition III.2: Soient $G$ un groupe localement compact de module $\Delta_{G}, \beta$ une action continue de $G$ sur $A, \psi$ dans $P(A)$. Les assertions suivantes sont équivalentes:

(i) $\psi$ est relativement invariant par rapport à $\beta$, de module $\Delta_{G}^{\prime}$ (voir [20]. Def 5.1), c'est-à-dire: $\psi \circ \beta_{s}=\Delta_{G}^{-1}(s) \psi(\forall s \in G)$.

(ii) $\psi$ est $\hat{\Delta}^{-1}$-relativement invariant par rapport à l'action $\beta^{d}$ de $K A(G)$ ([5], 8.1.1 et [4], I.3).

Démonstration: Comme, dans le cas où $\boldsymbol{K}=K A(G), \hat{\Delta}$ est l'opérateur modulaire associé au poids de Haar de $K A(G)^{\wedge}=K S(G)([5], 8.1 .4(\mathrm{c})$ ), c'est à dire la multiplication dans $L^{2}(G)$ par la fonction $\Delta_{G}$ ([5]. 8.1.7), l'implication (ii) $\Rightarrow$ (i) est claire.

Réciproquement, supposons (i); soient $f$ dans $L^{1}(G)^{+}$et $x$ dans $A^{+}$. On a:

$$
\int_{G} f(s) \psi\left(\beta_{s}(x)\right) d s=\psi(x) \int_{G} f(s) \Delta_{G}(s)^{-1} d s
$$

ce qui s'écrit encore, en considérant $L^{1}(G)$ comme le prédual de $L^{\infty}(G)$ :

$$
(\psi \otimes f)\left(\beta^{d}(x)\right)=\psi(x)\left\langle\Delta^{-1}, f\right\rangle
$$

d'où, par définition du poids opératoriel $\psi \otimes i$, la condition (i) de III.1.

Soit maintenant $f$ dans $L^{2}(G)$ telle que $\Delta_{G}^{1 / 4} f$ et $\hat{\Delta}_{G}^{-1 / 4} f$ appartiennent à $L^{2}(G)$. On a alors, pour $x$ et $y$ dans $\mathfrak{\Re}_{\psi}$ : 


$$
\begin{aligned}
\int_{G} \psi\left(\beta_{s}(y)^{*} x\right) \Delta_{G}(s)^{1 / 2}|f(s)|^{2} d s & =\int_{G} \psi\left(\beta_{s}\left(y \beta\left({ }_{-s}(x)\right)\right) \Delta_{G}(s)^{1 / 2}|f(s)|^{2} d s\right. \\
& =\int_{G} \psi\left(y^{*} \beta_{-s}(x)\right) \Delta_{G}(s)^{-1 / 2}|f(s)|^{2} d s
\end{aligned}
$$

par hypothèse d'où la condition (ii) de III.1.

Proposition 1II.3: Le poids de Haar $\varphi$ est $\hat{\Delta}^{-1}$-relativement invariant par rapport à l'action $\Gamma$.

Démonstration: Cela résulte de I.3 et I.14.

Théorème III.4: On suppose $\alpha$ intégrable. Soit $\psi$ dans $P\left(A^{\alpha}\right)$. Le poids $\psi \circ T_{\alpha}$ appartient à $P(A)$ et il est $\hat{\Delta}^{-1}$-relativement invariant par rapport à $\alpha$.

Démonstration: Par hypothèse (II.3) $T_{\alpha}$ est normal, semi-fini et fidèle, donc $\psi \circ T_{a} \in P(A)$ ([9], Prop. 2.3.).

De plus, pour tout $x$ dans $A^{+}$et $\xi$ dans $\mathscr{D}\left(\hat{\Delta}^{-1 / 2}\right)$, on a:

$$
\begin{aligned}
\left(\psi \circ T_{\alpha} \otimes \omega_{\xi}\right)(\alpha(x)) & =\left(\psi \otimes \omega_{\xi}\right)\left(\left(T_{\alpha} \otimes i\right)(\alpha(x))\right. \\
& =\left(\psi \otimes \omega_{\xi}\right)((i \otimes \varphi) \alpha \otimes i)(\alpha(x)) \\
& =\left(\psi \otimes \omega_{\xi}\right)(i \otimes \varphi \otimes i)(i \otimes \Gamma)(\alpha(x)) \\
& =\left(\psi \otimes \varphi \otimes \omega_{\xi}\right)(i \otimes \Gamma)(\alpha(x)) \\
& =(\psi \otimes \varphi)(\alpha(x))\left\|\hat{\Delta}^{-1 / 2} \xi\right\|^{2} \quad \text { d'après I.4. } \\
& =\psi(i \otimes \varphi)(\alpha(x))\left\|\hat{\Delta}^{-1 / 2} \xi\right\|^{2} \\
& =\psi \circ T_{\alpha}(x)\left\|\hat{\Delta}^{-1 / 2} \xi\right\|^{2}
\end{aligned}
$$

Soit $x$ dans $\mathfrak{M}_{\psi \circ{ }^{\circ} \alpha}^{+}$. On sait ([9], lemme 1.4.) qu'il existe un sous-espace fermé $H^{\prime}$ de $H$, et un opérateur autoadjoint positif $T$ sur $H^{\prime}$, tels que $\mathscr{D}(T)^{-}=H^{\prime}$ et

$$
\begin{aligned}
\omega_{\eta}\left(\left(\psi \circ T_{\alpha} \otimes i\right)(\alpha(x))\right) & =\left\|T^{1 / 2} \eta\right\|^{2} & & \text { si } \eta \in \mathscr{D}\left(T^{1 / 2}\right) \\
& =+\infty & & \text { sinon. }
\end{aligned}
$$

Il résulte de ce qui précède que $\mathscr{D}\left(T^{1 / 2}\right)$ contient $\mathscr{D}\left(\Delta^{-1 / 2}\right)$ dense dans $H$, donc que $H^{\prime}=H$.

De plus pour tout $\xi$ de $\mathscr{D}\left(\hat{\Delta}^{-1 / 2}\right)$, on a:

$$
\left\|T^{1 / 2} \xi\right\|^{2}=\psi \circ T_{\alpha}(x)\left\|\hat{\Delta}^{-1 / 2} \xi\right\|^{2} .
$$

Par l'unicité de la décomposition polaire, il en résulte:

$$
T^{1 / 2}=\psi \circ T_{\alpha}(x)^{1 / 2} \hat{\Delta}^{-1 / 2}
$$


et donc

$$
T=\psi \circ T_{\alpha}(x) \hat{\Delta}^{-1}
$$

finalement

$$
\left(\psi \circ T_{a} \otimes i\right)(\alpha(x))=\psi \circ T_{\alpha}(x) \hat{\Delta}^{-1} \quad \forall(x) \in \mathfrak{M}_{\psi \circ T a}^{+}
$$

d'où la condition (i) de III.1,. par normalité.

Soient $x$ et $y$ dans $\mathfrak{N}_{\psi_{\circ} T_{a}}$ (alors $\alpha(x)$ et $\alpha(y)$ appartiennent à $\mathfrak{N}_{\psi \otimes \varphi}$ ) et $\xi$ dans $\mathscr{D}\left(\hat{\Delta}^{1 / 4}\right) \cap \mathscr{D}\left(\hat{\Delta}^{-1 / 4}\right)$. Alors:

$$
\begin{aligned}
\left(\psi \circ T_{\alpha} \otimes \omega_{\hat{\Delta}^{1 / 4} \xi}\right)\left(\alpha\left(y^{*}\right)(x \otimes 1)\right)=\left(\psi \otimes \varphi \otimes \omega_{\hat{\Delta}^{1 / 4} \xi}\right)\left((\alpha \otimes i)\left(\alpha\left(y^{*}\right)(x \otimes 1)\right)\right) \\
\quad=\left(\psi \otimes \varphi \otimes \omega_{\hat{\Delta}^{1 / 4} \xi}\right)\left((i \otimes \Gamma)\left(\alpha\left(y^{*}\right)\right)(\alpha(x) \otimes 1)\right) \\
\quad=\left(\psi \otimes \psi \otimes \omega_{\left.\hat{\Delta}^{1 / 4} \xi \circ \kappa\right)\left(\left(\alpha\left(y^{*}\right) \otimes 1\right)(i \otimes \Gamma)(\alpha(x))\right)} \quad\right. \text { d'après I.13. } \\
\quad=\left(\psi \otimes \varphi \otimes \omega_{\left.\hat{\Delta}^{-1 / 4} \xi \circ \kappa\right)\left((\alpha \otimes i)\left(\left(y^{*} \otimes 1\right) \alpha(x)\right)\right)}\right. \\
\quad=\left(\psi \circ T_{a} \otimes \omega_{\hat{\Delta}^{-1 / 4} \xi} \circ \kappa\right)\left(\left(y^{*} \otimes 1\right) \alpha(x)\right)
\end{aligned}
$$

d'où la condition (ii) de III.1.

Définition HII.5: On dira que le quadruplet $(\mathcal{H}, \rho, \mathcal{G}, U)$, où $\mathscr{H}$ est un espace hilbertien, $\rho$ une représentation normale fidèle de $A$ sur $\mathscr{H}, \mathcal{G}$ un opérateur involutif, isométrique et antilinéaire sur $\mathscr{H}$ et $U$ un opérateur unitaire de $\mathcal{L}(\mathcal{H} \otimes H)$ est une forme canonique de $\alpha$ si:

(i) $\mathcal{I} \rho(A) \mathcal{G}=\rho(A)^{\prime}$

(ii) $U$ est un $1_{\boldsymbol{K}}^{\mathcal{L}(\mathscr{H})}$-cocycle qui implémente l'action $(\rho \otimes i) \alpha \rho^{-1} \operatorname{sur} \rho(A)$

(iii) $(\mathcal{I} \otimes \hat{J}) U(\mathscr{g} \otimes \hat{J})=U^{*}$.

Remarquons que (iii) signifie qu'à $\rho$ près, $U$ implémente $\alpha$ sur $A$.

Exemple III.6: (i) Soit $\beta$ une action continue d'un groupe localement compact $G$ sur $A$. Le quadruplet $\left(\mathscr{H}, \rho, \mathcal{g},\left(u_{s}\right)_{s \in G}\right)$ est une forme canonique de $\beta^{d}$ si:

$$
\mathscr{g} \rho(A) \mathscr{J}=\rho(A)^{\prime}
$$

l'application $s \rightarrow u_{s}$ est une représentation unitaire continue de $G$ sur $\mathscr{H}$ telle que $u_{s} \rho(x) u_{s}^{*}=\rho\left(\beta_{s}(x)\right)$.

$$
g u_{s}=u_{s} g \quad(\forall s \in G)
$$

(ii) le quadruplet $(H, i, J, \sigma(\hat{J} \otimes \hat{J}) W(\hat{J} \otimes \hat{J}) \sigma)$ est une forme canonique de l'action $\Gamma$.

Dans ce qui suit on suppose que le poids $\psi$ est $\hat{\Delta}^{-1}$ relativement invariant 
par rapport à $\alpha$.

De plus, pour simplifier les notations, on identifie $A$ et $\pi_{\psi}(A)$, autrement dit on considère $A \subset \mathcal{L}\left(H_{\psi}\right)$.

Lemme III.7: (i) Il existe une unique isométrie $U_{\psi}$ telle que, pour tous $x$ de $\mathfrak{N}_{\psi}$ et $y$ de $\mathfrak{N}_{\varphi} \cap \mathfrak{N}_{\varphi \circ_{\kappa}}$, on ait:

$$
U_{\psi}\left(\Lambda_{\psi}(x) \otimes \Delta^{-1 / 2} \Lambda_{\varphi}(y)\right)=\Lambda_{\psi \otimes \varphi}(\alpha(x)(1 \otimes y))
$$

(ii) $U_{\psi} \in \mathcal{L}\left(H_{\psi}\right) \otimes M$

(iii) $\alpha(a) U_{\psi}=U_{\psi}(\alpha \otimes 1)$.

Démonstration: D'après [5], 2.3.2.1., $\Lambda_{\varphi}(y)$ appartient à $\mathscr{D}\left(\hat{\Delta}^{-1 / 2}\right)$ et donc grâce à IIII.1. (ii), on a:

$$
\left(\psi \otimes \omega_{\Lambda_{\varphi}(y)}\right)\left(\alpha\left(x^{*} x\right)\right)=\psi\left(x^{*} x\right)\left\|\hat{\Delta}^{-1 / 2} \Lambda_{\varphi}(y)\right\|^{2} .
$$

Donc $\alpha\left(x^{*} x\right)$ appartient à $\mathfrak{M}_{\psi \otimes_{\omega_{\Lambda}(y)}}$ : en utilisant [17], II.11. il vient alors:

$$
\left(\psi \otimes \omega_{\Lambda_{\varphi}(y)}\right)\left(\alpha\left(x^{*} x\right)\right)=(\psi \otimes \varphi)\left(\left(1 \otimes y^{*}\right) \alpha\left(x^{*} x\right)(1 \otimes y)\right) .
$$

Ainsi $\alpha(x)(1 \otimes y)$ appartient à $\mathfrak{N}_{\psi \otimes \varphi}$ et

$$
\left\|\Lambda_{\psi \otimes \varphi}(\alpha(x)(1 \otimes y))\right\|^{2}=\left\|\Lambda_{\psi}(x)\right\|^{2}\left\|\hat{\Delta}^{-1 / 2} \Lambda_{\varphi}(y)\right\|^{2} .
$$

Comme $\Lambda_{\psi}\left(\mathfrak{\Re}_{\psi}\right) \otimes \hat{\Delta}^{-1 / 2} \Lambda_{\varphi}\left(\mathfrak{N}_{\psi} \cap \mathfrak{R}_{\varphi \circ \kappa}\right)$ est dense dans $H_{\psi \otimes \varphi}$, on en déduit (i) par polarisation.

Soit $\xi$ dans l'algèbre hilbertienne à droite $\mathfrak{U}_{\psi}^{\prime}$ associée au poids $\psi$. On notera $\pi_{\psi}^{\prime}(\xi)$ le prolongement continu à $H_{\psi}$ de la multiplication à droite par $\xi$ dans $\mathfrak{U}_{\psi}^{\prime}$. Soit $\eta$ dans $\mathfrak{u}^{\prime}$. On a:

$$
\begin{aligned}
\left(\pi_{\psi}^{\prime}(\xi) \otimes \pi^{\prime}(\eta)\right) U_{\psi}\left(\Lambda_{\psi}(x) \otimes \hat{\Delta}^{-1 / 2} \Lambda_{\varphi}(y)\right) & =\left(\pi_{\psi}^{\prime}(\xi) \otimes \pi^{\prime}(\eta)\right) \Lambda_{\psi \otimes \varphi}(\alpha(x)(1 \otimes y)) \\
& =\alpha(x)(1 \otimes y)(\xi \otimes \eta) \\
& =\alpha(x)(\xi \otimes y \eta) \\
& =\alpha(x)\left(1 \otimes \pi^{\prime}(\eta)\right)\left(\xi \otimes \Lambda_{\varphi}(y)\right) \\
& =\left(1 \otimes \pi^{\prime}(\eta)\right) \alpha(x)\left(\xi \otimes \Lambda_{\varphi}(y)\right) .
\end{aligned}
$$

En faisant tendre fortement $\pi^{\prime}(\pi)$ vers 1 , on obtient:

$$
\left(\pi_{\psi}^{\prime}(\xi) \otimes 1\right) U_{\psi}\left(\Lambda_{\psi}(x) \otimes \hat{\Delta}^{-1 / 2} \Lambda_{\varphi}(y)\right)=\alpha(x)\left(\xi \otimes \Lambda_{\varphi}(y)\right)
$$

Comme $\Lambda_{\varphi}\left(\mathfrak{N}_{\varphi} \cap \mathfrak{N}_{\varphi_{\circ \kappa}}\right)$ est un domaine essentiel pour $\hat{\Delta}^{-1 / 2}([5]$, lemme 3.1.2. (b)) on en déduit: 


$$
\left(\pi_{\psi}^{\prime}(\xi) \otimes 1\right) U_{\psi}\left(\Lambda_{\sigma}(x) \otimes \hat{\Delta}^{-1 / 2} \zeta\right)=\alpha(x)(\xi \otimes \zeta), \quad \forall \zeta \in \mathscr{D}\left(\hat{\Delta}^{-1 / 2}\right)(*) .
$$

Soit alors $z$ dans, $M^{\prime}$ comme $\hat{\Delta}^{-1 / 2}$ est affilié au centre de $M, z \zeta$ appartient à $\mathscr{D}\left(\hat{\Delta}^{-1 / 2}\right)$ et $\hat{\Delta}^{-1 / 2} z \zeta=z \hat{\Delta}^{-1 / 2} \zeta . \quad D^{\prime}$ où :

$$
\begin{aligned}
\left(\pi_{\psi}^{\prime}(\xi)\right. & \otimes 1) U_{\psi}(1 \otimes z)\left(\Lambda_{\psi}(x) \otimes \hat{\Delta}^{-1 / 2} \zeta\right) \\
& =\left(\pi_{\psi}^{\prime}(\xi) \otimes 1\right) U_{\psi}\left(\Lambda_{\psi}(x) \otimes \hat{\Delta}^{-1 / 2} z \zeta\right) \\
& =\alpha(x)(\xi \otimes z \zeta) \quad \text { d'après }(*) \\
& =\alpha(x)(1 \otimes z)(\xi \otimes \zeta) \quad \\
& =(1 \otimes z) \alpha(x)(\xi \otimes \zeta) \\
& =(1 \otimes z)\left(\pi_{\psi}^{\prime}(\xi) \otimes 1\right) U_{\psi}\left(\Lambda_{\psi}(x) \otimes \hat{\Delta}^{-1 / 2} \zeta\right) \quad \text { d'après } \quad(*) .
\end{aligned}
$$

En faisant tendre fortement $\pi_{\psi}^{\prime}(\xi)$ vers 1 , il vient:

$$
U_{\psi}(1 \otimes z)\left(\Lambda_{\psi}(x) \otimes \hat{\Delta}^{-1 / 2} \zeta\right)=(1 \otimes z) U_{\psi}\left(\Lambda_{\psi}(x) \otimes \hat{\Delta}^{-1 / 2} \zeta\right) .
$$

Par linéarité et densité, on en déduit:

$$
U_{\psi}(1 \otimes z)=(1 \otimes z) U_{\psi}, \quad \text { d'où (ii) . }
$$

Soit a dans $A$. On a:

$$
\begin{aligned}
U_{\psi}(a \otimes 1)\left(\Lambda_{\psi}(x) \otimes \hat{\Delta}^{-1 / 2} \Lambda_{\varphi}(y)\right) & =U_{\psi}\left(\Lambda_{\psi}(a x) \otimes \hat{\Delta}^{-1 / 2} \Lambda_{\varphi}(y)\right) \\
& =\Lambda_{\psi \otimes \varphi}(\alpha(a x)(1 \otimes y)) \\
& =\alpha(a) \Lambda_{\psi \otimes \varphi}(\alpha(x)(1 \otimes y)) \\
& =\alpha(a) U_{\psi}\left(\Lambda_{\psi}(x) \otimes \hat{\Delta}^{-1 / 2} \Lambda_{\varphi}(y)\right)
\end{aligned}
$$

d'où (iii) par linéarité et densité.

Lemme III.8: Soit $U_{\psi}$ l'isométrie construite en III.7.

(i) la relation $\mu(\omega)=(i \otimes \omega)\left(U_{\psi}^{*}\right)$, pour $\omega$ dans $M_{*}$ définit une application linéaire continue $\mu$ de $M_{*}$ dans $\mathcal{L}\left(H_{\psi}\right)$.

(ii) pour tous $\xi$, $\eta$ dans $H_{\psi}$ et $\zeta, \theta$ dans $H$, on $\alpha$ :

$$
\begin{aligned}
\left(\mu\left(\omega_{\zeta, \theta}\right) \xi \mid \eta\right) & =\left\langle U_{\psi}^{*}, \Omega_{\xi, \eta} \otimes \omega_{\zeta, \theta}\right\rangle \\
& =\left(\xi \otimes \zeta \mid U_{\psi}(\eta \otimes \theta)\right) .
\end{aligned}
$$

Démonstration: On a :

$$
\begin{aligned}
\|\mu(\omega)\| & =\sup \left\{\mid\langle\mu(\omega), \Omega\rangle, \Omega \in \mathcal{L}(H)_{*},\|\Omega\| \leqslant 1\right\} \\
& =\sup \left\{\left|(\Omega \otimes \omega)\left(U_{*}^{*}\right)\right|, \Omega \in \mathcal{L}(H)_{*},\|\Omega\| \leqslant 1\right\} \\
& \leqslant\|\omega\|, \quad \text { d'où (i). }
\end{aligned}
$$

La preuve de (ii) est triviale. 
Dans ce qui suit, on considère $U_{\psi}$ et $\mu$ tels qu'ils ont été définis en III.7 et III.8.

Lemme III.9: (i) $(i \otimes k)\left(U_{\psi}\right)=U_{\psi}^{*}$

(ii) Pour tout $\omega$ de $M_{*}$, on $a: \mu\left(\omega^{\circ}\right)=\mu(\omega)^{*}$.

Remarquons que pour $\xi$ dans $H$, grâce à [5], 1.2.2.1 et au rappel I.11, (ii) fournit $\mu\left(\omega_{\xi}\right)^{*}=\mu\left(\omega_{\hat{\jmath}}\right)$.

Démonstration: Soient $x$ et $y$ dans $\mathfrak{R}_{\varphi}$ et $\xi$ dans $\hat{\mathfrak{U}}_{\circ} \hat{\uparrow} \hat{\mathfrak{A}}_{\circ}$, de sorte que d'une part $\xi$ et $\hat{J} \xi$ appartiennent à $\underset{s \in C}{\cap} \mathscr{D}\left(\hat{\Delta}^{s}\right)$ et, d'autre part d'après [5] 3.1.2., $\pi(\xi)$ et $\pi(\hat{J} \xi)$ sont définis et appartiennent à $\mathfrak{N}_{\varphi} \cap \mathfrak{R}_{\varphi_{\text {o }_{\kappa}}}$. On a alors:

$$
\begin{aligned}
& \left\langle(i \otimes \kappa)\left(U_{\psi}\right), \Omega_{\Lambda_{\psi}(x), \Lambda_{\psi}(y)} \otimes \omega_{\xi}\right\rangle \\
& =\left\langle U_{\psi}, \Omega_{\Lambda_{\psi}(x), \Lambda_{\psi}(y)} \otimes \omega_{\xi} \circ \kappa\right\rangle \\
& =\left\langle U_{\psi}, \Omega_{\Lambda_{\psi}(x), \Lambda_{\psi}(y)} \otimes \omega \hat{\jmath}_{\xi}\right\rangle \quad \text { d'après le rappel I.11 } \\
& =\left(U_{\psi}\left(\Lambda_{\psi}(x) \otimes \hat{J} \xi\right) \mid \Lambda_{\psi}(y) \otimes \hat{J} \xi\right) \\
& =\left(\left(1 \otimes \hat{\Delta}^{1 / 2}\right) U_{\psi}\left(\Lambda_{\psi}(x) \otimes \hat{\Delta}^{-1 / 2} \hat{J} \xi\right) \mid \Lambda_{\psi}(y) \otimes \hat{J} \xi\right) \text { d'après III.7 (ii) et [5] 4.2.1. } \\
& =\left(\left(1 \otimes \hat{\Delta}^{1 / 2}\right) \Lambda_{\psi \otimes \varphi}(\alpha(x)(1 \otimes \pi(\hat{J} \xi))) \mid \Lambda_{\psi}(y) \otimes \hat{J} \xi\right) \quad \text { d'après III.7 (i). } \\
& =\lim _{\varepsilon \rightarrow 0}\left(\left(1 \otimes \hat{\Delta}^{1 / 2}\right)_{\varepsilon} \Lambda_{\psi \otimes \varphi}(\alpha(x)(1 \otimes \pi(\hat{J} \xi))) \mid \Lambda_{\psi}(y) \otimes \hat{J} \xi\right) \\
& =\lim _{\varepsilon \rightarrow 0}(\psi \otimes \varphi)\left(\left(1 \otimes \hat{\Delta}^{1 / 2}\right)_{\varepsilon}\left(y^{*} \otimes \pi(\hat{J} \xi)^{*}\right) \alpha(x)(1 \otimes \pi(\hat{J} \xi))\right) \\
& =\lim _{\varepsilon \rightarrow 0}(\psi \otimes \omega \hat{\jmath} \xi)\left(\left(1 \otimes \hat{\Delta}^{1 / 2}\right)_{\varepsilon}\left(y^{*} \otimes 1\right) \alpha(x)\right) \quad \text { grâce à [17], II.11. } \\
& =\left(\psi \otimes \omega_{\hat{J} \xi}\left(\hat{\Delta}^{1 / 2} \cdot\right)\right)\left(\left(y^{*} \otimes 1\right) \alpha(x)\right) \quad \text { grâce à }(\mathbb{P} 4) \\
& =\left(\psi \otimes\left(\omega_{\xi} \circ \kappa\right)\left(\hat{\Delta}^{1 / 2} \cdot\right)\right)\left(\left(y^{*} \otimes 1\right) \alpha(x)\right) \quad \text { d'après le rappel I.11 } \\
& =\left(\psi \otimes \omega_{\left.\hat{\Delta}^{-1 / 4} \xi^{\circ} \circ \kappa\right)}\right)\left(\left(y^{*} \otimes 1\right) \alpha(x)\right) \quad \text { d'après } \mathrm{I} .12 \\
& =\left(\psi \otimes \omega_{\hat{\Delta}^{1 / 4} \xi}\right)\left(\alpha\left(y^{*}\right)(x \otimes 1)\right) \quad \text { d'après III.1 (ii) } \\
& =\lim _{\varepsilon \rightarrow 0}\left(\psi \otimes \omega_{\xi}\right)\left(\left(1 \otimes \hat{\Delta}^{1 / 2}\right)_{\varepsilon} \alpha\left(y^{*}\right)(x \otimes 1)\right) \\
& =\lim _{\varepsilon \rightarrow 0}(\psi \otimes \varphi)\left(\left(1 \otimes \hat{\Delta}^{1 / 2}\right)_{\varepsilon}\left(1 \otimes \pi(\xi)^{*}\right) \alpha\left(y^{*}\right)(x \otimes \pi(\xi))\right) \quad \text { grâce à [17], II.11. } \\
& \left.=\lim _{\varepsilon \rightarrow 0}\left(\left(1 \otimes \Delta^{1 / 2}\right)_{\varepsilon}\left(\Lambda_{\psi}(x)\right) \otimes \xi\right) \mid \Lambda_{\psi \otimes \varphi}(\alpha(y)(1 \otimes \pi)(\xi))\right) \\
& =\lim _{\varepsilon \rightarrow 0}\left(\left(1 \otimes \hat{\Delta}^{1 / 2}\right)_{\varepsilon}\left(\Lambda_{\psi}(x) \otimes \xi\right) \mid U_{\psi}\left(\Lambda_{\psi}(y) \otimes \hat{\Delta}^{-1 / 2} \xi\right)\right) \quad \text { d'après III.7. (i) } \\
& =\left(\Lambda_{\psi}(x) \otimes \xi \mid U_{\psi}\left(\Lambda_{\psi}(y) \otimes \xi\right)\right) \quad \text { d'après III.7. (ii) et [5] 4.2.1. } \\
& =\left\langle U_{\psi}^{*}, \Omega_{\Lambda_{\psi}(x), \Lambda_{\psi}(y)} \otimes \omega_{\xi}\right\rangle
\end{aligned}
$$

d'où (i), par densité.

Soit $\omega$ dans $M_{*}^{+}$. D'après [5], 1.2.2.1., on a: 


$$
\begin{array}{rlrl}
\mu\left(\omega^{\circ}\right) & =\mu(\omega \circ \kappa) & & \\
& =(i \otimes \omega \circ \kappa)\left(U_{\psi}^{*}\right) & & \\
& =(i \otimes \omega)\left(U_{\psi}\right) & & \text { d'après (i) } \\
& =\left((i \otimes \omega)\left(U_{\psi}^{*}\right)\right)^{*} & & \text { car } \omega \text { est positif } \\
& =\mu(\omega)^{*} . &
\end{array}
$$

Oon en déduit (ii) par antilinéarité

Lemme III.10: Soint $x$ dans $\mathfrak{\Re}_{\psi}$ et $\xi$ dans $\hat{\mathfrak{A}}_{\circ} \hat{\uparrow}_{\hat{\mathfrak{U}}}$. Alors $\left(i \otimes \omega_{\hat{\Delta}^{1 / 4} \xi}\right)(\alpha(x))$ appartient a $\mathfrak{N}_{\psi}$ et $\Lambda_{\psi}\left(\left(i \otimes \omega_{\hat{\Delta}^{1 / 4} \xi}\right)(\alpha(x))\right)=\mu\left(\omega_{\xi}\right)^{*} \Lambda_{\psi}(x)$.

Démonstration: Supposons que $\left\|\hat{\Delta}^{1 / 4} \xi\right\|=1$; alors $i \otimes \omega_{\hat{\Delta}^{1 / 4} \xi}$ est une espérance conditionnelle et, d'après [21]:

$\left(\left(i \otimes \omega_{\hat{\Delta}^{1 / 4} \xi}\right)(\alpha(x))\right)^{*}\left(i \otimes \omega_{\hat{\Delta}^{1 / 4} \xi}\right)(\alpha(x)) \leqslant\left(i \otimes \omega_{\hat{\Delta}^{1 / 4} \xi}\right)\left(\alpha\left(x^{*} x\right)\right)$

d'où

$\psi\left(\left(\left(i \otimes \omega_{\hat{\Delta}^{1 / 4}}\right)(\alpha(x))\right) *\left(i \otimes \omega_{\hat{\Delta}^{-1 / 4}}\right)(\alpha(x))\right) \leqslant\left(\psi \otimes \omega_{\hat{\Delta}^{1 / 4} \xi}\right)\left(\alpha\left(x^{*} x\right)\right)$

$$
=\psi\left(x^{*} x\right)\left\|\hat{\Delta}^{-1 / 4} \xi\right\|^{2} \quad \text { d'après III.1(i). }
$$

d'où, par linéarité, la première partie du lemme.

Soit $y$ dans $\mathfrak{N}_{\psi}$. On a:

$$
\begin{array}{rlr}
\left(\Lambda_{\psi}\left(\left(i \otimes \omega_{\hat{\Delta}^{1 / 4} \xi}\right)(\alpha(x))\right) \mid \Lambda_{\psi}(y)\right) & =\psi\left(y^{*}\left(i \otimes \omega_{\hat{\Delta}^{-1 / 4}}\right)(\alpha(x))\right) & \\
& =\psi\left(\left(i \otimes \omega_{\hat{\Delta}^{1 / 4} \xi}\right)\left(\left(y^{*} \otimes 1\right) \alpha(x)\right)\right) & \text { d'après [21] } \\
& =\left(\psi \otimes \omega_{\hat{\Delta}^{1 / 4} \xi}\right)\left(\left(y^{*} \otimes 1\right) \alpha(x)\right) & \text { grâce à [17], } 0.4 . \\
& =\lim _{\varepsilon \rightarrow 0}\left(\psi \otimes \omega_{\xi}\right)\left(\left(1 \otimes \hat{\Delta}^{1 / 2}\right)_{\varepsilon}\left(y^{*} \otimes 1\right) \alpha(x)\right) & \\
& =\lim _{\varepsilon \rightarrow 0}(\psi \otimes \varphi)\left(\left(1 \otimes \hat{\Delta}^{1 / 2}\right)_{\varepsilon}\left(y^{*} \otimes \pi(\xi)^{*}\right) \alpha(x)(1 \otimes \pi(\xi))\right. \\
& =\lim _{\varepsilon \rightarrow 0}\left(\Lambda_{\psi \otimes \varphi}(\alpha(x)(1 \otimes \pi(\xi))) \mid\left(1 \otimes \hat{\Delta}^{1 / 2}\right)_{\varepsilon}\left(\Lambda_{\psi}(y) \otimes \xi\right)\right) \\
& =\lim _{\varepsilon \rightarrow 0}\left(U_{\psi}\left(\Lambda_{\psi}(x) \otimes \hat{\Delta}^{-1 / 2} \xi\right) \mid\left(1 \otimes \hat{\Delta}^{1 / 2}\right)_{\varepsilon}\left(\Lambda_{\psi}(y) \otimes \xi\right)\right) \\
& =\left(U_{\psi}\left(\Lambda_{\psi}(x) \otimes \xi\right) \mid \Lambda_{\psi}(y) \otimes \xi\right) & \text { d'après III.7 (ii) } \\
& =\left(\mu\left(\omega_{\xi}\right) \Lambda_{\psi}(y) \mid \Lambda_{\psi}(x)\right)^{-} & \text {d'après III.7 (i) } \\
& =\left(\mu\left(\omega_{\xi}\right)^{*} \Lambda_{\psi}(x) \mid \Lambda_{\psi}(y)\right) &
\end{array}
$$


d'où le lemme, par linéarité et densité.

Lemme III.11: (i) Pour tout $\omega$ de $M_{*}$ et $t$ de Re, $\mu(\omega)$ commute à $\Delta_{\psi}^{i} t$ $\left(\Delta_{\psi}\right.$ est l'opérateur modulaire associe au poids $\left.\psi\right)$.

(ii) Pour tout $\omega$ de $M_{*}^{+}, \mu(\omega)$ commute à $J_{\psi}\left(J_{\psi}\right.$ est l'involution antilinéaire isométrique associée à $\psi$ ).

(iii) Pour tout $t$ de $\boldsymbol{R}$, on $a:\left(\Delta_{\psi}^{i t} \otimes 1\right) U_{\psi}=U_{\psi}\left(\Delta_{\psi}^{i t} \otimes 1\right)$

Démonstration: Soient $x$ dans $\mathfrak{N}_{\psi} \cap \mathfrak{R}_{\psi}^{*}$ et $\xi$ dans $\hat{\mathfrak{A}}_{\circ} \hat{\top}_{\hat{\mathfrak{U}}} . \quad \mathbb{I}$ résulte $\mathrm{du}$ lemme précédent que $\mu\left(\omega_{\xi}\right) * \Lambda_{\psi}(x)=\Lambda_{\psi}\left(\left(i \otimes \omega_{\hat{\Delta}^{1 / 4} \xi}\right)(\alpha(x))\right)$ appartient à $\Lambda_{\psi}\left(\mathfrak{N}_{\psi} \cap \mathfrak{R}_{\psi}^{*}\right)$ et donc au domaine de $S_{\psi}$ (prolongement fermé de l'application $\Lambda_{\psi}(x) \rightarrow \Lambda_{\psi} /\left(x^{*}\right)$ définie sur $\left.\Lambda_{\psi}\left(\mathfrak{R}_{\psi} \cap \mathfrak{R}_{\psi}^{*}\right)\right)$. De plus:

$$
\begin{aligned}
S_{\psi} \mu\left(\omega_{\xi}\right)^{*} \Lambda_{\psi}(x) & =\Lambda_{\psi}\left(\left(i \otimes \omega_{\Delta^{1 / 4} \xi}\right)\left(\alpha\left(x^{*}\right)\right)\right) \\
& =\mu\left(\omega_{\xi}\right)^{*} \Lambda_{\psi}\left(x^{*}\right) \\
& =\mu\left(\omega_{\xi}\right)^{*} S_{\psi} \Lambda_{\psi}(x)
\end{aligned}
$$

par suite, comme $\Lambda_{\psi}\left(\mathfrak{N}_{\psi} \cap \mathfrak{N}_{\psi}^{*}\right)$ est un domaine essentiel pour $S_{\psi}$ :

$$
\mu\left(\omega_{\xi}\right) * S_{\psi} \subset S_{\psi} \mu\left(\omega_{\xi}\right)^{*} .
$$

Le vecteur $\hat{J} \xi$ vérifie les mêmes hypothèses que $\xi$; on en déduit donc (IIII.9 (ii)) que:

$$
\mu\left(\omega_{\xi}\right) S_{\psi} \subset S_{\psi} \mu\left(\omega_{\xi}\right)
$$

D'autre part, en transposant $(*)$ il vient:

$$
\begin{aligned}
& \mu\left(\omega_{\xi}\right) F_{\psi} \subset F_{\psi} \mu\left(\omega_{\xi}\right) \quad \text { et: } \\
& \mu\left(\omega_{\xi}\right) \Delta_{\psi}=\mu\left(\omega_{\xi}\right) F_{\psi} S_{\psi} \subset F_{\psi} \mu\left(\omega_{\xi}\right) S_{\psi} \subset F_{\psi} S_{\psi} \mu\left(\omega_{\xi}\right)=\Delta_{\psi} \mu\left(\omega_{\xi}\right)
\end{aligned}
$$

d'où

$$
\mu\left(\omega_{\xi}\right) \Delta_{\psi}^{i t}=\Delta_{\psi}^{i t} \mu\left(\omega_{\xi}\right) \quad\left(\forall t \in \mathbb{R}, \forall \xi \in \mathfrak{U} \circ \hat{\uparrow} \mathfrak{U}_{\circ}\right)
$$

et (i) en résulte par linéarité et continuité (cf. III.8 (ii)).

On a également

$$
\begin{array}{rlrl}
\mu\left(\omega_{\xi}\right) J_{\psi} \Lambda_{\psi}(x) & =\mu\left(\omega_{\xi}\right) \Delta_{\psi}^{1 / 2} S_{\psi} \Lambda_{\psi}(x) & \\
& =\Delta_{\psi}^{1 / 2} \mu\left(\omega_{\xi}\right) S_{\psi} \Lambda_{\psi}(x) & & \text { d'après ce qui précède } \\
& =\Delta_{\psi}^{1 / 2} S_{\psi} \mu\left(\omega_{\xi}\right) \Lambda_{\psi}(x) & & \text { d'après }(* *) \\
& =J_{\psi} \mu\left(\omega_{\xi}\right) \Lambda_{\psi}(x) &
\end{array}
$$

et (ii) s'en déduit par densité et continuité. 
Soient $\xi_{1}, \xi_{2}$ dans $H_{\psi}$ et $\eta, \zeta$ dans $H$. On a:

$$
\begin{array}{rlrl}
\left(\xi_{1} \otimes \eta \mid\left(\Delta_{\psi}^{i t} \otimes 1\right) U_{\psi}\left(\xi_{2} \otimes \zeta\right)\right) & =\left(\Delta_{\psi}^{-i t} \xi_{1} \otimes \eta \mid U_{\psi}\left(\xi_{2} \otimes \zeta\right)\right) & & \\
& =\left(\mu\left(\omega_{\eta, \zeta}\right) \Delta_{\psi}^{-i t} \xi_{1} \mid \xi_{2}\right) & & \text { d'après III.8 (ii) } \\
& =\left(\Delta_{\psi}^{-i t} \mu\left(\omega_{\eta, \zeta}\right) \xi_{1} \mid \xi_{2}\right) & & \text { d'après (i) } \\
& =\left(\mu\left(\omega_{\eta, \zeta}\right) \xi_{1} \mid \Delta_{\psi}^{i t} \xi_{2}\right) & \\
& =\left(\xi_{1} \otimes \eta \mid U_{\psi}\left(\Delta_{\psi}^{i t} \xi_{2} \otimes \zeta\right)\right) & & \text { d'après III.8 (ii). } \\
& =\left(\xi_{1} \otimes \eta \mid U_{\psi}\left(\Delta_{\psi}^{i t} \otimes 1\right)\left(\xi_{2} \otimes \zeta\right)\right)
\end{array}
$$

d'où (iii) par linéarité, densité et continuité.

Lemme III.12: $\quad\left(J_{\psi} \otimes \hat{J}\right) U_{\psi}\left(J_{\psi} \otimes \hat{J}\right)=U_{\psi}^{*}$

Démonstration: Soient $\xi$ dans $H$ et $\eta, \zeta$ dans $H_{\psi}$. On a:

$$
\begin{array}{rlrl}
\left(\left(J_{\psi} \otimes \hat{J}\right) U_{\psi}\left(J_{\psi} \otimes \hat{J}\right)(\eta \otimes \xi) \mid \zeta \otimes \xi\right) & =\left(J_{\psi} \zeta \otimes \hat{J} \xi \mid U_{\psi}\left(J_{\psi} \eta \otimes \hat{J} \xi\right)\right) \\
& =\left(\mu\left(\omega_{\hat{J} \xi}\right) J_{\psi} \zeta \mid J_{\psi} \eta\right) & & \text { d'après III.8 (ii) } \\
& =\left(\eta \mid \mu\left(\omega_{J \xi}\right) \zeta\right) & & \text { d'après III.11 (ii) } \\
& =\left(\eta \mid \mu\left(\omega_{\xi}\right)^{*}\right) \zeta & & \text { grâce à III.9 (ii). } \\
& =\left(\mu\left(\omega_{\xi}\right) \eta \mid \zeta\right) & & \\
& =\left(\eta \otimes \xi \mid U_{\psi}(\zeta \otimes \xi)\right) & & \text { d'après III.8 (ii) } \\
& =\left(U_{\psi}^{*}(\eta \otimes \xi) \mid \zeta \otimes \xi\right) &
\end{array}
$$

d'où le résultat, par polarisation, linéarité et continuité.

Corollaire III.13: (i) $U_{\psi}$ est unitaire

(ii) $\alpha(\alpha)=U_{\psi}(\alpha \otimes 1) U_{\psi}^{*} \quad(\forall a \in A)$

(iii) $\alpha \sigma_{t}^{\psi}=\left(\sigma_{t}^{\psi} \otimes i\right) \alpha \quad(\forall t \in \boldsymbol{R})$.

Démonstration: (i) résulte trivialement de III.12; (ii) de III.7. (iii) et de (i); et (iii) de III.11. (iii) et de (ii).

Lemme III.14: Soient a dans $\mathfrak{N}_{\psi \otimes \varphi}, y$ dans $\mathfrak{\Re}_{\varphi} ;$ alors $(i \otimes \Gamma)(a)(1 \otimes y \otimes 1)$ appartient à $\mathfrak{N}_{\psi \otimes \varphi \otimes \varphi}$ et :

$$
\Lambda_{\psi \otimes \varphi \otimes \varphi}((i \otimes \Gamma)(a)(1 \otimes y \otimes 1))=(1 \otimes W)(1 \otimes \sigma)\left(\Lambda_{\psi \otimes \varphi}(a) \otimes \Lambda_{\varphi}(y)\right) .
$$

Démonstration: Soient $a_{1}$ dans $\mathfrak{N}_{\psi}$ et $a_{2}$ dans $\mathfrak{N}_{\varphi}$. On a:

$$
\begin{aligned}
(i \otimes \Gamma)\left(a_{1} \otimes a_{2}\right)(1 \otimes y \otimes 1) & =a_{1} \otimes \Gamma\left(a_{2}\right)(y \otimes 1) \\
& \in \mathfrak{R}_{\psi} \otimes \mathfrak{N}_{\varphi \otimes \varphi} \quad \text { d'après [5], 2.1.1. }
\end{aligned}
$$

et 


$$
\begin{aligned}
\Lambda_{\psi \otimes \varphi \otimes \varphi}\left(a_{1} \otimes \Gamma\left(a_{2}\right)(y \otimes 1)\right) & =\Lambda_{\psi}\left(a_{1}\right) \otimes W\left(\Lambda_{\varphi}(y) \otimes \Lambda_{\varphi}\left(a_{2}\right)\right) \quad \text { d'après [5], 2.1.1. } \\
& =(1 \otimes W)(1 \otimes \sigma)\left(\Lambda_{\psi}\left(a_{1}\right) \otimes \Lambda_{\psi}\left(a_{2}\right) \otimes \Lambda_{\varphi}(y)\right) \quad(*) .
\end{aligned}
$$

Considérons sur $A \otimes M$ les deux poids normaux définis par

$$
\begin{aligned}
& \Phi(b)=(\psi \otimes \varphi)(b) \varphi\left(y^{*} y\right) \\
& \Psi(b)=(\psi \otimes \varphi \otimes \varphi)\left(\left(1 \otimes y^{*} \otimes 1\right)(1 \otimes \Gamma)(b)(1 \otimes y \otimes 1)\right)
\end{aligned}
$$

Il en clair que $\Phi$ est semi-fini et fidèle, il résulte de ce qui précède que $\Psi$ est semi-fini. Il résulte de [5] 2.2.5. (c) que $\Psi$ est invariant par $\sigma=\sigma^{\psi} \otimes \sigma^{\varphi}$; comme $W$ est unitaire, le lemme 4.1.1. de [5] (avec $E=\mathfrak{N}_{\psi} \otimes \mathfrak{N}_{\varphi}$ ) permet de conclure que $\Phi=\Psi$.

Donc, si a appartient à $\mathfrak{N}_{\psi \otimes \varphi},(i \otimes \Gamma)(a)(1 \otimes y \otimes 1)$ appartient à $\mathfrak{N}_{\psi \otimes \varphi \otimes \varphi}$ et $\left\|\Lambda_{\psi \otimes \varphi \otimes \varphi}((i \otimes \Gamma)(a)(1 \otimes y \otimes 1))\right\|=\left\|\Lambda_{\psi \otimes \varphi \otimes \varphi}(a \otimes y)\right\|$.

Ainsi il existe une isométrie de $H_{\psi \otimes \varphi \otimes \varphi}$ dans lui-même qui envoie $\Lambda_{\psi \otimes \varphi \otimes \varphi}(a \otimes y)$ sur $\Lambda_{\psi \otimes \varphi \otimes \varphi}((i \otimes \Gamma)(a)(1 \otimes y \otimes 1))$; il résulte de (*) qu'elle coincide avec $(1 \otimes W)(1 \otimes \sigma)$ sur $\Lambda_{\psi}\left(\mathfrak{N}_{\psi}\right) \otimes \Lambda_{\varphi}\left(\mathfrak{N}_{\varphi}\right) \otimes \Lambda_{\varphi}\left(\mathfrak{R}_{\varphi}\right)$, d'où le lemme

Lemme III.15: Soit $a$ dans $\mathfrak{N}_{\psi \otimes \varphi}, z$ dans $\mathfrak{R}_{\varphi} \cap \mathfrak{\Re}_{\varphi_{\circ \kappa} \text {. }} \quad$ Alors $(\alpha \otimes i)(a)$. $(1 \otimes z \otimes 1)$ appartient $a \mathfrak{N}_{\psi \otimes \varphi \otimes \varphi}$ et

$$
\Lambda_{\psi \otimes \varphi \otimes \varphi}((\alpha \otimes i)(a)(1 \otimes z \otimes 1))=\left(1 \otimes \Delta^{-1 / 2} \otimes 1\right)\left(U_{\psi} \otimes 1\right)(1 \otimes \sigma) \Lambda_{\psi \otimes \varphi \otimes \varphi}(a \otimes z)
$$

Démonstration: Soient $a_{1}$, dans $\mathscr{I}_{\psi}$ et $a_{2}$ dans $\mathscr{T}_{\varphi}$. II résulte de III.7 (i) que

$$
\begin{aligned}
\Lambda_{\psi \otimes \varphi \otimes \varphi}\left(\alpha\left(a_{1}\right)(1 \otimes z) \otimes a_{2}\right) & =U_{\psi}\left(\Lambda_{\psi}\left(a_{1}\right) \otimes \Delta^{-1 / 2} \Lambda_{\varphi}(z)\right) \otimes \Lambda_{\varphi}\left(a_{2}\right) \\
& =\left(U_{\psi} \otimes 1\right)(1 \otimes \sigma)\left(\Lambda_{\psi}\left(a_{1}\right) \otimes \Lambda_{\varphi}\left(a_{2}\right) \otimes \Delta^{-1 / 2} \Lambda_{\varphi}(z)\right) .
\end{aligned}
$$

Considérons sur $A \otimes M$ les deux poids normaux définis par:

$$
\begin{aligned}
& \Phi(b)=(\Psi \otimes \varphi)(b) \varphi \circ \kappa\left(z^{*} z\right) \\
& \Psi(b)=(\psi \otimes \varphi \otimes \varphi)\left(\left(1 \otimes z^{*} \otimes 1\right)(\alpha \otimes i)(b)(1 \otimes z \otimes 1)\right)
\end{aligned}
$$

Comme il résulte de III.13 (iii) que $\Psi$ est invariant par $\sigma=\sigma^{\Psi} \otimes \sigma^{\varphi}$ et que $\Delta$ est la dérivée de Radon-Nikodym de $\varphi$ par rapport à $\varphi \circ \kappa([5], 4.2 .1$.) en utilisant [5] 4.1.1. (avec $E=\mathfrak{R}_{\psi} \otimes\left(\mathfrak{R}_{\varphi} \cap \mathfrak{R}_{\varphi \text { o }_{k}}\right)$ ). On prouve que $\Phi=\Psi$

Ainsi-t-on:

$$
\left.\left(U_{\psi} \otimes 1\right)\right)(1 \otimes \sigma)\left(\Lambda_{\psi \otimes \varphi}(a) \otimes \Delta^{-1 / 2} \Lambda_{\varphi}(z)\right)=\Lambda_{\psi \otimes \varphi \otimes \varphi}((\alpha \otimes i)(\alpha)(1 \otimes z \otimes 1))
$$

et le lemme résulte de III.7 (ii) et [5] 4.2.1. 
Lemme III.16: Soient $x$ dans $\mathfrak{N}_{\varphi}$ et $\xi$ dans $\Lambda_{\varphi \otimes \varphi}\left(\mathfrak{N}_{\varphi \otimes \varphi}\right) \cap \mathscr{D}\left(\Delta^{-1 / 2} \otimes \Delta^{-1 / 2}\right)$. Alors $((\alpha \otimes i) \alpha(x))(1 \otimes(\pi \otimes \pi)(\xi))$ appartient à $\mathfrak{R}_{\psi \otimes \varphi \otimes \varphi}$ et

$$
\begin{aligned}
& \Lambda_{\psi \otimes \varphi \otimes \varphi}(((\alpha \otimes i) \alpha(x))(1 \otimes(\pi \otimes \pi)(\xi))) \\
& \quad=\left(1 \otimes \Delta^{-1 / 2} \otimes \Delta^{-1 / 2}\right)\left(U_{\psi} \otimes 1\right)(1 \otimes \sigma)\left(U_{\psi} \otimes 1\right)(1 \otimes \sigma)\left(\Lambda_{\psi}(x) \otimes \xi\right) .
\end{aligned}
$$

Démonstration: Soient $a_{1}$ et $a_{2}$ dans $\mathfrak{N}_{\varphi} \cap \mathfrak{N}_{\varphi_{0_{\kappa} \kappa}} . \quad$ On a $\alpha(x)\left(1 \otimes a_{2}\right) \in$ $\mathfrak{\Re}_{\psi \otimes \varphi}$ d'après III.7 (i), et il résulte de III.15 que:

$$
\begin{aligned}
\Lambda_{\psi \otimes \varphi \otimes \varphi}( & \left.(\alpha \otimes i)\left(\alpha(x)\left(1 \otimes a_{2}\right)\right)\left(1 \otimes a_{1} \otimes 1\right)\right)= \\
= & \left(U_{\psi} \otimes 1\right)(1 \otimes \sigma)_{\psi \otimes \varphi}\left(\alpha(x)\left(1 \otimes a_{2}\right)\right) \otimes \Delta^{-1 / 2} \Lambda_{\varphi}\left(a_{1}\right) \\
= & \left(U_{\psi} \otimes 1\right)(1 \otimes \sigma)\left(U_{\varphi} \otimes 1\right)\left(\Lambda_{\psi}(x) \otimes \Delta^{-1 / 2} \Lambda_{\varphi}\left(a_{2}\right) \otimes \Delta^{-1 / 2} \Lambda_{\varphi}\left(a_{1}\right)\right) \\
\quad & \text { d'après III.7 (i) } \\
= & \left(U_{\psi} \otimes 1\right)(1 \otimes \sigma)\left(U_{\psi} \otimes 1\right)(1 \otimes \sigma)\left(\Lambda_{\psi}(x) \otimes \Delta^{-1 / 2} \Lambda_{\varphi}\left(a_{1}\right) \otimes \Delta^{-1 / 2} \Lambda_{\varphi}\left(a_{2}\right)\right) .
\end{aligned}
$$

Soient $\eta$ dans $\mathfrak{U}_{\varphi \otimes \varphi}^{\prime}$ et $\zeta$ dans $\mathfrak{X}_{\psi}^{\prime}$. On a donc:

$$
\begin{aligned}
\left(\pi_{\psi}^{\prime}(\zeta)\right. & \left.\otimes(\pi \otimes \pi)^{\prime}(\eta)\right)\left(U_{\psi} \otimes 1\right)(1 \otimes \sigma)\left(U_{\psi} \otimes 1\right)(1 \otimes \sigma)\left(\Lambda_{\psi}(x) \otimes \hat{\Delta}^{-1 / 2} \Lambda_{\varphi}\left(a_{1}\right) \otimes \Delta^{-1 / 2} \Lambda_{\varphi}\left(a_{2}\right)\right) \\
& =(\alpha \otimes i)(\alpha(x))\left(1 \otimes a_{1} \otimes a_{2}\right)(\zeta \otimes \eta) \\
& =(\alpha \otimes i)(\alpha(x))\left(\left(1 \otimes(\pi \otimes \pi)^{\prime}(\eta)\right)\left(\zeta \otimes \Lambda_{\varphi \otimes \varphi}\left(a_{1} \otimes a_{2}\right)\right) .\right.
\end{aligned}
$$

On peut choisir $a_{1}$ et $a_{2}$ de telle sorte que $\Lambda_{\varphi \otimes \varphi}\left(a_{1} \otimes a_{2}\right)$ tende vers $\xi$ et $\Delta^{-1 / 2} \Lambda_{\varphi}\left(a_{1}\right) \otimes \Delta^{-1 / 2} \Lambda_{\psi}\left(a_{2}\right)$ tende vers $\left(\Delta^{-1 / 2} \otimes \Delta^{-1 / 2}\right) \xi$ (cf. [5] 3.1.2 (b)). On obtient:

$$
\begin{aligned}
\left(\pi_{\psi}^{\prime}(\zeta)\right. & \left.\otimes(\pi \otimes \pi)^{\prime}(\eta)\right)\left(U_{\psi} \otimes 1\right)(1 \otimes \sigma)\left(U_{\psi} \otimes 1\right)(1 \otimes \sigma)\left(\Lambda_{\psi}(x) \otimes\left(\hat{\Delta}^{-1 / 2} \otimes \hat{\Delta}^{-1 / 2}\right) \xi\right) \\
& =(\alpha \otimes i)(\alpha(x))\left(\left(1 \otimes(\pi \otimes \pi)^{\prime}(\eta)\right)(\zeta \otimes \xi)\right) \\
& =(\alpha \otimes i)(\alpha(x))((1 \otimes(\pi \otimes \pi)(\xi))(\zeta \otimes \eta)) .
\end{aligned}
$$

Donc, le vecteur $\left(U_{\psi} \otimes 1\right)(1 \otimes \sigma)\left(U_{\psi} \otimes 1\right)(1 \otimes \sigma)\left(\Lambda_{\psi}(x) \otimes\left(\Delta^{-1 / 2} \otimes \Delta^{-1 / 2}\right) \xi\right)$ est borné à gauche par rapport à $\psi \otimes \varphi \otimes \varphi$ et l'on a:

$$
\begin{aligned}
& \left(\pi_{\psi} \otimes \pi \otimes \pi\right)\left(\left(U_{\psi} \otimes 1\right)(1 \otimes \sigma)\left(U_{\psi} \otimes 1\right)(1 \otimes \sigma)\left(\Lambda_{\psi}(x) \otimes\left(\Delta^{-1 / 2} \otimes \Delta^{-1 / 2}\right) \xi\right)\right)= \\
& =(\alpha \otimes i)(\alpha(x))(1 \otimes(\pi \otimes \pi)(\xi))
\end{aligned}
$$

Le lemme résulte alors de III.7 (iii) et [5] 4.2.1.

\section{Lemme III.17:}

$(1 \otimes W)(1 \otimes \sigma)\left(U_{\psi} \otimes 1\right)(1 \otimes \sigma)=\left(U_{\psi} \otimes 1\right)(1 \otimes \sigma)\left(U_{\psi} \otimes 1\right)(1 \otimes \sigma)(1 \otimes W)$

Démonstration: Soient $x$ dans $\mathfrak{N}_{\psi}, y$ dans $\mathfrak{N}_{\varphi}, z$ dans $\mathfrak{N}_{\varphi} \cap \mathfrak{N}_{\varphi_{\mathrm{o}} \mathrm{K}}$. On a: 
$(1 \otimes W)(1 \otimes \sigma)\left(U_{\psi} \otimes 1\right)(1 \otimes \sigma) \Lambda_{\psi \otimes \varphi \otimes \varphi}(x \otimes y \otimes z)$

$=(1 \otimes W)(1 \otimes \sigma)\left(U_{\psi} \Lambda_{\psi \otimes \varphi}(x \otimes z) \otimes \Lambda_{\varphi}(y)\right)$

$=(1 \otimes W)(1 \otimes \sigma)\left(\left(1 \otimes \Delta^{1 / 2}\right) U_{\psi}\left(\Lambda_{\psi}(x) \otimes \Delta^{-1 / 2} \Lambda_{\psi}(z)\right) \otimes \Lambda_{\varphi}(y)\right)$

d'après III.7 (ii) et [5], 4.2.1.

$=(1 \otimes W)(1 \otimes \sigma)\left(1 \otimes \Delta^{1 / 2} \otimes 1\right)\left(\Lambda_{\psi \otimes \varphi}(\alpha(x)(1 \otimes z)) \otimes \Lambda_{\varphi}(y)\right) \quad$ d'après III.7 (i)

$=\left(1 \otimes \Delta^{1 / 2} \otimes \Delta^{1 / 2}\right)(1 \otimes W)(1 \otimes \sigma)\left(\Lambda_{\psi \otimes \varphi}(\alpha(x)(1 \otimes z)) \otimes \Lambda_{\varphi}(y)\right) \quad$ d'après I.1.

$=\left(1 \otimes \Delta^{1 / 2} \otimes \Delta^{1 / 2}\right) \Lambda_{\psi \otimes \varphi \otimes \varphi}((i \otimes \Gamma)(\alpha(x)(1 \otimes z))(1 \otimes y \otimes 1)) \quad$ d'après III.14

$=\left(1 \otimes \Delta^{1 / 2} \otimes \Delta^{1 / 2}\right) \Lambda_{\psi \otimes \varphi \otimes \varphi}((\alpha \otimes i)(\alpha(x))(1 \otimes \Gamma(z)(y \otimes 1)))$

$=\left(U_{\psi} \otimes 1\right)(1 \otimes \sigma)\left(U_{\psi} \otimes 1\right)(1 \otimes \sigma)\left(\Lambda_{\psi}(x) \otimes \Lambda_{\varphi \otimes \varphi}(\Gamma(z)(y \otimes 1))\right) \quad$ d'après I.16 et III.16

$=\left(U_{\psi} \otimes 1\right)(1 \otimes \sigma)\left(U_{\psi} \otimes 1\right)(1 \otimes \sigma)(1 \otimes W)\left(\Lambda_{\psi}(x) \otimes \Lambda_{\varphi}(y) \otimes \Lambda_{\varphi}(z)\right)$

d'après [5] 2.1.1.

d'où le résultat.

Théorème 11I.18: Le quadruplet $\left(H_{\psi}, \pi_{\psi}, J_{\psi}, U_{\psi}\right)$ est une forme canonique de $\alpha$.

Démonstration: Il résulte du lemme précédent que:

$(1 \otimes W)(1 \otimes \sigma)\left(U_{\psi} \otimes 1\right)(1 \otimes \sigma)\left(1 \otimes W^{*}\right)=\left(U_{\psi} \otimes 1\right)(1 \otimes \sigma)\left(U_{\psi} \otimes 1\right)(1 \otimes \sigma)$

Soit, d'après [4], I.5 (iii) et III.7 (ii)

$$
(i \otimes \Gamma)\left(U_{\psi}\right)=\left(U_{\psi} \otimes 1\right)(1 \otimes \sigma)\left(U_{\psi} \otimes 1\right)(1 \otimes \sigma)
$$

Le théorème résulte alors de III.12 et III.13 (i) et (ii).

Corollaire III.19: On suppose $\alpha$ integrable. Soit $\psi^{\prime}$ dans $P(A)$. Alors il existe un unitaire $U$ dans $\mathcal{L}\left(H_{\psi^{\prime}}\right) \otimes M$ tel que $\left(H_{\psi^{\prime}}, \pi_{\psi^{\prime}}, J_{\psi^{\prime}}, U\right)$ soit une forme canonique de $\alpha$.

Démonstration: D'après III.4, il existe un poids $\psi$ sur $A, \hat{\Delta}^{-1}$ relativement invariant par rapport à $\alpha$. D'après le théorème précédent, il existe $U_{\psi}$ tel que $\left(H_{\psi}, \pi_{\psi}, J_{\psi}, U_{\psi}\right)$ soit une forme canonique de $\alpha$. Soit alors $V$ l'unitaire $H_{\psi} \rightarrow H_{\psi^{\prime}}$ construit comme en [8], 2.18. Posons $U=(V \otimes 1) U_{\psi}\left(V^{*} \otimes 1\right)$; le résultat se déduit alors aisément de [8], 2.18.

\section{Propriétés des produits croisés}

On utilise les notations du chapitre II. 
Lemme IV.1: (i) On suppose que $\alpha$ admet une forme canonique ( $\mathcal{H}, \rho$, g, $U$ ). Alors si a vérifie $(A)$, elle vérifie $(B)$.

(ii) Toute action duale vérifie $(B)$.

Démonstration: On pose $\beta=(\rho \otimes i) \alpha \rho^{-1}$. On suppose que $\alpha$ vérifie $(A)$, il résulte alors de [4], III.10 que $\beta$ également. Autrement dit:

$$
\left(\beta(\rho(A)) \cup \boldsymbol{C}_{\mathscr{l}} \otimes \mathcal{L}(H)\right)^{\prime \prime}=\rho(A) \otimes \mathcal{L}(H)
$$

soit encore:

$$
\left(U\left(\rho(A) \otimes \boldsymbol{C}_{H}\right) U^{*} \cup \boldsymbol{C}_{\mathscr{H}} \otimes \mathcal{L}(H)\right)^{\prime \prime}=\rho(A) \otimes \mathcal{L}(H)
$$

d'où, d'après III.5. (iv):

$$
\left((\mathscr{g} \otimes \hat{J}) U^{*}(\mathscr{g} \otimes \hat{J})\left(\rho(A) \otimes \boldsymbol{C}_{H}\right)(\mathscr{g} \otimes \hat{J}) U(\mathscr{g} \otimes \hat{J}) \cup \boldsymbol{C}_{\mathscr{H}} \otimes \mathcal{L}(H)\right)^{\prime \prime}=\rho(A) \otimes \mathcal{L}(H)
$$

et, grâce à III.5. (ii):

$$
\left(U^{*}\left(\rho(A)^{\prime} \otimes C_{H}\right) U \cup C_{\mathscr{H}} \otimes \mathcal{L}(H)\right)^{\prime \prime}=\rho(A)^{\prime} \otimes \mathcal{L}(H) .
$$

Ainsi, l'action $\beta^{\prime}$ implémentée par $U^{*}$ sur $\rho(A)^{\prime}$ (II.7) vérifie $(A)$; donc $\beta$ vérifie (B) (II.13) et $\alpha$ également (II.6. (ii)); ce qui achève la démonstration de (i).

D'après [4], III 5., toute action duale vérifie $(A)$ et d'après II.4 est intégrable, donc admet une forme canonique (III.19). Ainsi (ii) résulte de (i).

Théorème IV.2: (i) Toute action vérifie $(A)$

(ii) Toute action vérifie $(B)$

(iii) Toute action est saturée

Démonstration: D'après le lemme précédent, l'action biduale $\widetilde{\widetilde{\alpha}}$ vérifie (B). D'autre part, d'action $r$ introduite en II.15. (i) est équivalente à $\widetilde{\widetilde{\alpha}}$ (II.15. (iii)), elle vérifie donc $(B)$ (II.6. (ii)).

Or, par définition:

$$
\begin{aligned}
\mathscr{W}^{*}(r) & =\left((i \otimes \varsigma)(\alpha \otimes i)\left(\alpha(A) \cup C_{K} \otimes \mathcal{L}(H)\right)^{\prime \prime} \cup C_{K} \otimes H\right. \\
& =\left((i \otimes \varsigma)\left((\alpha \otimes i)(\alpha(A)) \cup \hat{M}^{\prime}\right)^{\prime \prime}\right. \\
& \left.=(i \otimes \zeta)\left((\alpha \otimes i)(\alpha(A)) \cup C_{K} \otimes \hat{M}^{\prime} \otimes \mathcal{L}(H)\right)^{\prime \prime} \cup C_{K} \otimes_{H} \otimes \hat{M}^{\prime}\right)^{\prime \prime} \\
& =(i \otimes \varsigma)\left(\left((\alpha \otimes i)(\alpha(A)) \cup C_{K} \otimes \hat{M}^{\prime} \otimes M\right)^{\prime \prime} \cup C_{K}{ }_{H} \otimes \mathcal{L}(H)\right)^{\prime \prime} \\
& =(i \otimes \varsigma)\left(\mathscr{W}^{*}(\alpha) \otimes M \cup C_{K} \otimes H\right. \\
& =(i \otimes \varsigma)\left(\mathscr{W}^{*}(\alpha) \otimes \mathcal{L}(H)\right)^{\prime \prime} \quad \text { d'après II.14. }
\end{aligned}
$$

Et donc, d'après II.6. (i): 


$$
\begin{aligned}
\mathscr{W}^{*}(r)^{\tilde{\gamma}} & =\mathscr{W}^{*}(r) \cap \mathcal{L}(K \otimes H) \otimes M \\
& =(i \otimes \varsigma)(\mathscr{W} *(\alpha) \otimes \mathcal{L}(H) \cap \mathcal{L}(K) \otimes M \otimes \mathcal{L}(H)) \\
& =(i \otimes \varsigma)\left(\left(\mathscr{W}^{*}(\alpha) \cap \mathcal{L}(K) \otimes M\right) \otimes \mathcal{L}(H)\right) \\
& =(i \otimes \varsigma)\left(\mathscr{W}^{*}(\alpha)^{\tilde{\alpha}} \otimes \mathcal{L}(H)\right) \quad \text { grâce à II.6.(i). }
\end{aligned}
$$

Donc, comme $r$ vérifie $(\mathrm{B})$, on $\mathrm{a}$ :

$$
\begin{aligned}
(i \otimes \zeta)\left(\mathscr{W} *(\alpha)^{\tilde{\alpha}} \otimes \mathcal{L}(H)\right) & =r\left(\left(\alpha(A) \cup \boldsymbol{C}_{K} \otimes \mathcal{L}(H)\right)^{\prime \prime}\right) \\
& =(i \otimes \varsigma)(\alpha \otimes i)\left(\alpha(A) \cup \boldsymbol{C}_{K} \otimes \mathcal{L}(H)\right)^{\prime \prime}
\end{aligned}
$$

d'où :

$$
\begin{aligned}
\mathscr{Q}^{* *}(\alpha)^{\alpha} \otimes \mathcal{L}(H) & =(\alpha \otimes i)\left(\alpha(A) \cup \boldsymbol{C}_{K} \otimes \mathcal{L}(H)\right)^{\prime \prime} \\
& \subset(\alpha \otimes i)(A \otimes \mathcal{L}(H)) \\
& =\alpha(A) \otimes \mathcal{L}(H)
\end{aligned}
$$

ainsi :

$\mathscr{W}^{*}(\alpha)^{\tilde{\alpha}} \subset \alpha(A)$, d'où (ii), d'après [4], II.6.

La relation $(*)$ s'écrit alors:

$$
\alpha(A) \otimes \mathcal{L}(H)=(\alpha \otimes i)\left(\alpha(A) \cup \boldsymbol{C}_{K} \otimes \mathcal{L}(H)\right)^{\prime \prime}
$$

comme $\alpha \otimes i$ est injectif, on en déduit (i).

Supposons $\alpha$ implémentée par un $1_{\boldsymbol{K}}^{\mathcal{L}(K)}$-cocycle $U$; d'après (i) l'action $\alpha^{\prime}$ de $\mathbb{K}^{s}$ sur $A^{\prime}$ implémentée par $U^{*}$ vérifie $(A) ; \alpha$ est donc saturée (II.13).

Supposons $\alpha$ quelconque; il résulte de [4], I.13. que la restriction de $(\alpha \otimes i)$ à $\alpha(A)$ est une action implémentée et donc saturée. On a donc:

$$
\begin{aligned}
& (\alpha \otimes i)(\alpha(A))=\{x \in \alpha(A) \otimes M ;(\alpha \otimes i \otimes i)(x)=(i \otimes i \otimes \Gamma)(x)\} \\
& \quad=\{(\alpha \otimes i)(y) ; y \in A \otimes M,(\alpha \otimes i \otimes i)(\alpha \otimes i)(y)=(i \otimes i \otimes \Gamma)(\alpha \otimes i)(y)\} \\
& \quad=\{(\alpha \otimes i)(y) ; y \in A \otimes M,(\alpha \otimes i \otimes i)(\alpha \otimes i)(y)=(\alpha \otimes i \otimes i)(i \otimes \Gamma)(y)\} \\
& \quad=\{(\alpha \otimes i) y ; y \in A \otimes M,(\alpha \otimes i)(y)=(i \otimes \Gamma)(y)\}
\end{aligned}
$$

d'où (iii). Grâce à (iii) on obtient alors immédiatement (ii) en utilisant II.13.

Théorème IV.3 (Bidualité): Toute action $\alpha$ est telle que:

(i) $(i \otimes \varsigma)(\alpha \otimes i)$ est une action de $\mathbb{K}$ sur $A \otimes \mathcal{L}(H)$

(ii) $1 \otimes \sigma W^{*} \sigma$ est un $(i \otimes \varsigma)(\alpha \otimes i)$-cocycle

(iii) $\widetilde{\alpha} \sim(i \otimes \varsigma)(\alpha \otimes i)\left(\Theta(\alpha \otimes i), v, 1 \otimes \sigma W^{*} \sigma\right)$

(pour les définitions de $\Theta$ et $v$, voir lemme II.15). 
Démonstration: Cela résulte de [4], II.8 et IV.2 (i).

Ce résultat généralise [20], 4.5, [13] th 2 et 3, [14], 7.1.

Lemme $\mathbb{I V} .4: \quad$ La restriction de $\Theta(\alpha \otimes i)$ à $\mathscr{W}^{*}(\alpha)$ est égale à $\tilde{\alpha}$.

Démonstration: On a: $\quad \Theta(\alpha \otimes i) \alpha(x)=\alpha(x) \otimes 1 \quad$ pour $x$ dans $A$ et $\quad \Theta(1 \otimes 1 \otimes y)=1 \otimes \hat{\Gamma}^{\prime}(y) \quad$ pour $y$ dans $\hat{M}^{\prime}$

(voir le calcul fait en [4], III.1).

Il résulte donc de [4], II.6. (ii) que:

$$
\begin{array}{ll}
\Theta(\alpha \otimes i)(\alpha(x))=\alpha(\alpha(x)) & (\forall x \in A) \\
\Theta(\alpha \otimes i)(1 \otimes y)=\alpha(1 \otimes y) & \left(\forall y \in M^{\prime}\right)
\end{array}
$$

d'où le résultat, en appliquant [4], II.1.

Proposition $\mathbb{I V . 5 : ~ L ' a c t i o n ~} \beta$ de $\mathbb{K}$ sur $A \otimes \mathcal{L}(H)$ définie par

$$
\beta \sim(i \otimes \varsigma)(\alpha \otimes i) \quad\left(i, i, 1 \otimes \sigma W^{*} \sigma\right)
$$

vérifie: (i) $\widetilde{\widetilde{\alpha}} \approx \beta(\Theta(\alpha \otimes i), v)$

(ii) $(A \otimes \mathcal{L}(H))^{\beta}=\mathscr{W}^{*}(\alpha)$.

Démonstration: La définition de $\beta$ provient de IV.3. (i) et (ii) et de [4], I.8.

La relation (i) résulte de IV.3. (iii) en utilisant les formules de composition des équivalences ([4], I.11. 1 et 2).

Il résulte de II.16 que:

$(A \otimes \mathcal{L}(H))^{\beta}=\left\{x \in A \otimes \mathcal{L}(H) ; \Theta(\alpha \otimes i)(x) \in \mathscr{W} *(\widetilde{\alpha})^{\alpha \sim \sim}\right\}$, or en appliquant V.2. (ii) à $\widetilde{\alpha}$, on obtient $\mathscr{W} \mathcal{W}^{*}(\widetilde{\alpha})^{\alpha \sim \sim}=\widetilde{\alpha}\left(\mathscr{W} \mathcal{W}^{*}(\alpha)\right.$ ), d'où (ii), grâce à IV.4.

Corollaire IV.6: On suppose $\alpha$ implémentée par un $1_{\boldsymbol{K}}^{\mathcal{L}(\mathcal{K})}$-cocycle $U$. Alors:

$$
\mathscr{W}^{*}(\alpha)=A \otimes \mathcal{L}(H) \cap U\left(\mathcal{L}(K) \otimes \hat{M}^{\prime}\right) U^{*}
$$

Démonstration: On a:

$$
\begin{aligned}
\mathscr{W}^{*}(\alpha) & =(A \otimes \mathcal{L}(H))^{\beta} \quad \text { d'après IV.5. (ii) } \\
& =\left\{x \in A \otimes \mathcal{L}(H) ;(i \otimes \varsigma)(\alpha \otimes i)(x)=(1 \otimes \sigma W \sigma)(x \otimes 1)\left(1 \otimes \sigma W^{*} \sigma\right)\right\} \\
& \text { d'après II.16. }
\end{aligned}
$$

d'où le résultat, grâce à II.11. 
Théorème IV.7 (Commutation): On suppose $\alpha$ implémentée par un $1_{K}^{\mathcal{L}(K)}$-cocycle $U$; soit $\alpha^{\prime}$ l'action de $\mathbb{K}^{s}$ sur $A^{\prime}$ implémentée par $U^{*}$. Alors:

$$
\mathscr{W}^{*}(\alpha)^{\prime}=U \mathscr{W}^{*}\left(\alpha^{\prime}\right) U^{*}
$$

Démonstration: I1 résulte de IV.6. que:

et donc:

$$
\mathscr{W}^{*}(\alpha)^{\prime}=\left(A^{\prime} \otimes \mathbb{C}_{H} \cup U\left(\mathbb{C}_{K} \otimes \hat{M}\right) U^{*}\right)^{\prime \prime}
$$

$$
\begin{aligned}
U^{*} \mathscr{W}^{*}(\alpha)^{\prime} U & =\left(\alpha^{\prime}\left(A^{\prime}\right) \cup \mathbb{C}_{K} \otimes \hat{M}\right)^{\prime \prime} \\
& =\mathscr{W}^{*}\left(\alpha^{\prime}\right) \quad \text { par définition. }
\end{aligned}
$$

Cela généralise [3], th. 3.14. et [13], th. 5 .

Corollaire $\mathbf{L} .8:$ On suppose que a admet une forme canonique $(\mathcal{H}, \rho, \mathcal{G}, U)$. Alors $\left(\mathcal{H} \otimes H, \rho \otimes i, U(\mathscr{g} \otimes \hat{J}), 1 \otimes(J \hat{J} \otimes J \hat{J}) W^{*}(J \hat{J} \otimes J \hat{J})\right)$ est une forme canonique de $\widetilde{\alpha}$.

Démonstration: Comme $(\mathcal{H}, \rho, \mathcal{F}, U)$ est une forme canonique, on a: $U(\mathscr{g} \otimes \hat{J}) U(\mathscr{g} \otimes \hat{J})=U U^{*}=1$, donc l'opérateur $U(\mathscr{g} \otimes \hat{J})$ est involutif. Il est clair que $1 \otimes(J \hat{J} \otimes J \hat{J}) W^{*}(J \hat{J} \otimes J \hat{J})$ est un opérateur unitaire de $\mathcal{L}(\mathcal{H}) \otimes H \otimes H$.

Soit $\rho$ la représentation de $A$ sur $\mathscr{H}$ (III.5), il est clair que $\rho \otimes i$ est une représentation normale fidèle de $\mathscr{W}^{*}(\alpha)$ sur $\mathscr{H} \otimes H$.

On pose $\beta=(\rho \otimes i) \alpha \rho^{-1}$; cette action de $\mathbb{K}$ sur $\rho(A)$ est implémentée par $U$; on notera $\beta^{\prime}$ l'action de $\mathbb{K}^{s}$ sur $\rho(A)^{\prime}$ implémentée par $U^{*}$.

D'après [4], II.4, on a:

$$
\mathscr{W}^{*}(\beta)=(\rho \otimes i) \mathscr{W}^{*}(\alpha)
$$

Puis:

$$
\begin{aligned}
& U(\mathscr{G} \otimes \hat{J})(\rho \otimes i)(\mathscr{W} *(\alpha)) U(\mathscr{G} \otimes \hat{J}) \\
& =U(\mathscr{g} \otimes \hat{J})(\rho \otimes i) \mathscr{W} \mathcal{N}^{*}(\alpha)(\mathscr{g} \otimes \hat{J}) U^{*} \\
& =U(\mathscr{g} \otimes \hat{J}) \mathscr{W}^{*}(\beta)(\mathscr{g} \otimes \hat{J}) U^{*} \\
& =U(\mathscr{g} \otimes \hat{J})\left(U\left(\rho(A) \otimes C_{H}\right) U^{*} \cup C_{\mathscr{H}} \otimes \hat{M}\right)^{\prime \prime}(\mathscr{g} \otimes \hat{J}) U^{*} \\
& =U\left((\mathscr{g} \otimes \hat{J}) U\left(\rho(A) \otimes \mathbb{C}_{H}\right) U^{*}(\mathscr{g} \otimes \hat{J}) \cup \mathbb{C}_{\mathscr{H}} \otimes \hat{M}\right)^{\prime \prime} U^{*} \\
& =U\left(U^{*}\left(g \rho(A) \mathscr{g} \otimes C_{H}\right) U \cup \mathbb{C}_{\mathcal{H}} \otimes \hat{M}\right)^{\prime \prime} U^{*} \\
& =U\left(U^{*}\left(\rho(A)^{\prime} \otimes \mathbb{C}_{H}\right) U \cup \mathbb{C}_{\mathscr{l}} \otimes M\right)^{\prime \prime} U^{*} \\
& =U \mathscr{W}^{*}\left(\beta^{\prime}\right) U^{*} \\
& =\mathscr{W} *(\beta)^{\prime} \\
& =(\rho \otimes i)\left(\mathscr{W}^{*}(\alpha)\right)^{\prime} \\
& \text { d'après III.5. (iv) } \\
& \text { d'après }(*) \\
& \text { par définition } \\
& \text { d'après III.5. (iv) } \\
& \text { d'après III.5. (ii) } \\
& \text { par définition } \\
& \text { d'après IV.6. } \\
& \text { d'après }(*)
\end{aligned}
$$


d'où (i) de III.5.

D'autre part, [4], II.6. (i) énonce que $1 \otimes(J \hat{J} \otimes J \hat{J}) W^{*}(J \hat{J} \otimes J \hat{J})$ implémente $\widetilde{\beta}$ sur $\mathscr{W}^{*}(\beta)$; or, d'après [4], II.8., on a:

$$
\widetilde{\beta} \approx \widetilde{\alpha}(\rho \otimes i, i)
$$

soit $\tilde{\beta}=(\rho \otimes i \otimes i) \tilde{\alpha}(\rho \otimes i)^{-1}, \quad$ d'où (ii) de III.5.

Enfin, on a:

$$
\begin{aligned}
(U(g) \hat{J}) \otimes J)\left(1 \otimes(J \hat{J} \otimes J \hat{J}) W^{*}(J \hat{J} \otimes J \hat{J})(U(g \otimes \hat{J}) \otimes J)\right. \\
\quad=(U \otimes 1)(g \otimes J \otimes \hat{J})\left(1 \otimes W^{*}\right)(g \otimes J \otimes \hat{J})\left(U^{*} \otimes 1\right) \quad \text { d'après III.5. (iv) } \\
\quad=(U \otimes 1)(1 \otimes(J \hat{J} \otimes J \hat{J}) W(J \hat{J} \otimes J \hat{J}))\left(U^{*} \otimes 1\right) \quad \text { d'après [5], 3.1.5. (b) } \\
\quad=1 \otimes(J \hat{J} \otimes J \hat{J}) W(J \hat{J} \otimes J \hat{J})
\end{aligned}
$$

car $\quad U \otimes 1 \in \mathcal{L}(\mathscr{H}) \otimes M \otimes \boldsymbol{C}_{H}$

et $\quad 1 \otimes(J J \otimes J J) W(J J \otimes J J) \in \boldsymbol{C}_{\mathscr{H}} \otimes M^{\prime} \otimes \mathcal{L}(H) \quad$ d'après [5], 2.1.1.

d'où (iii) de III.5.

\section{Caractérisation des produits croisés}

On utilise les notations du chapitre II.

Lemme V.1: Soit $\mathscr{H}$ un espace hilbertien, $B$ une algébre de von Neumann sur $\mathcal{H}$. On suppose qu'il existe une action $\beta$ de $\hat{\boldsymbol{K}}^{\prime}$ sur $B$ et un morphisme normal $\nu$ de $\hat{M}^{\prime}$ sur B tel que:

$$
\begin{aligned}
& \nu(1)=1 \\
& \beta \nu=(\nu \otimes i) \hat{\Gamma}^{\prime} .
\end{aligned}
$$

Alors $Y=(\nu \otimes i)(\sigma(\hat{J} \otimes \hat{J}) W(\hat{J} \otimes \hat{J}) \sigma)$ est un $1_{\boldsymbol{K}}^{B}$-cocycle qui implémente une action de $\boldsymbol{K}$ sur $B^{\beta}$, qu'on notera $\delta$.

Démonstration: En faisant $\mathscr{H}=\boldsymbol{C}$ dans [4], I.13. (i), on voit que $\sigma(\hat{J} \otimes \hat{J}) W(\hat{J} \otimes \hat{J}) \sigma$ est un $1_{\boldsymbol{K}}^{\mathcal{L}(H)}$-cocycle. Plus précisément, comme cet opérateur appartient à $\hat{M}^{\prime} \otimes M\left([5], 2.1 .5\right.$. (b)), c'est un $1_{K}^{M^{\prime}}$-cocycle. On en déduit facilement que $Y$ est un $1_{\boldsymbol{K}}^{B}$-cocycle.

Calculons:

$(\beta \otimes i)(Y)$

$=(\beta \nu \otimes i)(\sigma(\hat{J} \otimes \hat{J}) W(\hat{J} \otimes \hat{J}) \sigma)$

$=(\nu \otimes i \otimes i)\left(\hat{\Gamma}^{\prime} \otimes i\right)(\sigma(\hat{J} \otimes \hat{J}) W(\hat{J} \otimes \hat{J}) \sigma)$

$=(\nu \otimes i \otimes i)((\sigma \otimes 1)(1 \otimes \sigma(\hat{J} \otimes \hat{J}) W(\hat{J} \otimes \hat{J}) \sigma)(\sigma \otimes 1)(1 \otimes \sigma(\hat{J} \otimes \hat{J}) W(\hat{J} \otimes \hat{J}) \sigma))$ 


d'après [17], II. 6
appliqué à $\hat{\mathbb{K}}^{\prime}(\mathrm{cf.}$ [17], III.14)
$=(\nu \otimes i \otimes i)((\varsigma \otimes i)(i \otimes \varsigma)(i \otimes(\hat{J} \otimes \hat{J}) W(\hat{J} \otimes \hat{J}))(1 \otimes \sigma(\hat{J} \otimes \hat{J}) W(\hat{J} \otimes \hat{J}) \sigma)$
$=(\nu \otimes i \otimes i)((i \otimes \varsigma)(\varsigma \otimes i)((\hat{J} \otimes \hat{J})(W(\hat{J} \otimes \hat{J}) \otimes 1))(1 \otimes \sigma(\hat{J} \otimes \hat{J}) W(\hat{J} \otimes \hat{J}) \sigma)$
$=(i \otimes \varsigma)((\nu \otimes i)(\sigma(\hat{J} \otimes \hat{J}) W(\hat{J} \otimes \hat{J}) \sigma \otimes 1)(1 \otimes(\hat{J} \otimes \hat{J}) W(\hat{J} \otimes \hat{J})))$
$=(i \otimes \varsigma)((Y \otimes 1)(1 \otimes(\hat{J} \otimes \hat{J}) W(\hat{J} \otimes \hat{J}))) \quad(*)$

Soit $x$ dans $B^{\beta}$. On a:

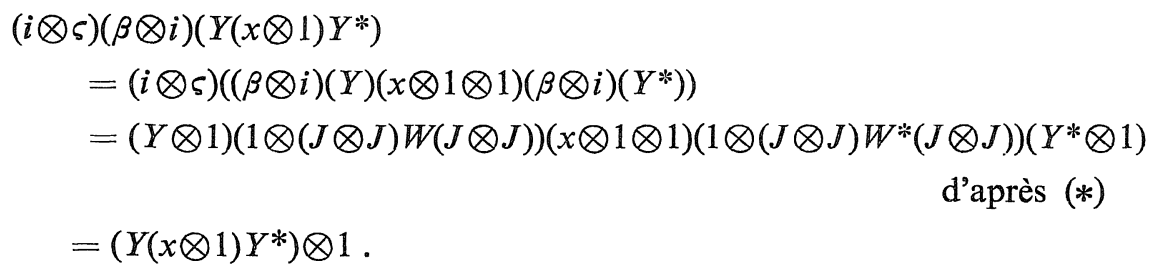

Ainsi $Y(x \otimes 1) Y^{*}$ appartient à $(B \otimes M)^{(i \otimes s)(\beta \otimes i)}=B^{\beta} \otimes M$, d'après II.17, d'où le résultat.

Théorème V.2: Soit B une algèbre de von Neumann. Les propositions suivantes sont équivalentes:

(i) il existe une action $\beta$ de $\hat{\mathbb{K}}^{\prime}$ sur $B$ et un morphisme normal $\nu$ de $\hat{M}^{\prime}$ dans $B$ tel que $\nu(1)=1$ et $\beta \nu=(\nu \otimes i) \hat{\Gamma}^{\prime}$

(ii) il existe une algèbre de von Neumann $C$, une action $\gamma$ de $\mathbb{K}$ soit $C$ telle que $B$ soit isomorphe à $\Psi^{*} *(\gamma)$.

(Ceci généralise [12], th. 1, [13], th. 1 et [14], th. 8.3.).

Démonstration: Supposons (ii), notons $\phi$ l'isomorphisme de $B$ sur $\mathscr{W} *(r)$. Il est clair que $\beta=\left(\phi^{-1} \otimes i\right) \tilde{\gamma} \phi$ et $\nu$ défini par $\nu(x)=\phi^{-1}(1 \otimes x)$ (pour $x$ dans $\hat{M}^{\prime}$ ) vérifient (i).

Réciproquement, supposons (i) et reprenons les notations de V.1.. Notons $\Psi$ le morphisme normal injectif (Ad $Y$ ) $\beta$ de $B$ dans $B \otimes \mathcal{L}(H)$. On a, par définition:

$$
\begin{aligned}
\mathscr{W}^{*}(\delta) & =\left(\delta\left(B^{\beta}\right) \cup \mathbb{C}_{\mathscr{H}} \otimes \hat{M}^{\prime}\right)^{\prime \prime} \\
& =\left((\operatorname{Ad} Y)\left(B^{\beta} \otimes \mathbb{C}_{H}\right) \cup \mathbb{C}_{\mathscr{H}} \otimes \hat{M}^{\prime}\right)^{\prime \prime} \\
& =(\operatorname{Ad} Y)\left(B^{\beta} \otimes \mathbb{C}_{H} \cup\left(\operatorname{Ad} Y^{*}\right)\left(\mathbb{C}_{\mathscr{H}} \otimes \hat{M}^{\prime}\right)^{\prime \prime}\right.
\end{aligned}
$$

Or, pour $x$ dans $\hat{M}^{\prime}$, on a: 


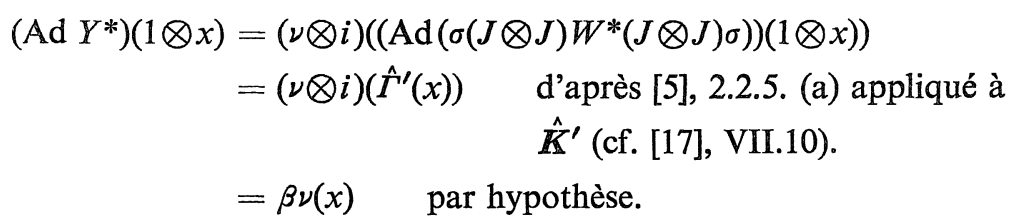

Il en résulte que:

$$
\begin{aligned}
\mathscr{W}^{*}(\delta) & =(\operatorname{Ad} Y)\left(B^{\beta} \otimes C_{\mathscr{G}} \cup \beta \nu\left(\hat{M}^{\prime}\right)\right)^{\prime \prime} \\
& =(\operatorname{Ad} Y) \circ \beta\left(B^{\beta} \cup \nu\left(\hat{M}^{\prime}\right)\right)^{\prime \prime} \\
& =\Psi\left(B^{\beta} \cup \nu\left(\hat{M}^{\prime}\right)\right)^{\prime \prime} \\
& \subset \Psi(B) \quad(*)
\end{aligned}
$$

Soit $y$ dans $B$. On a:

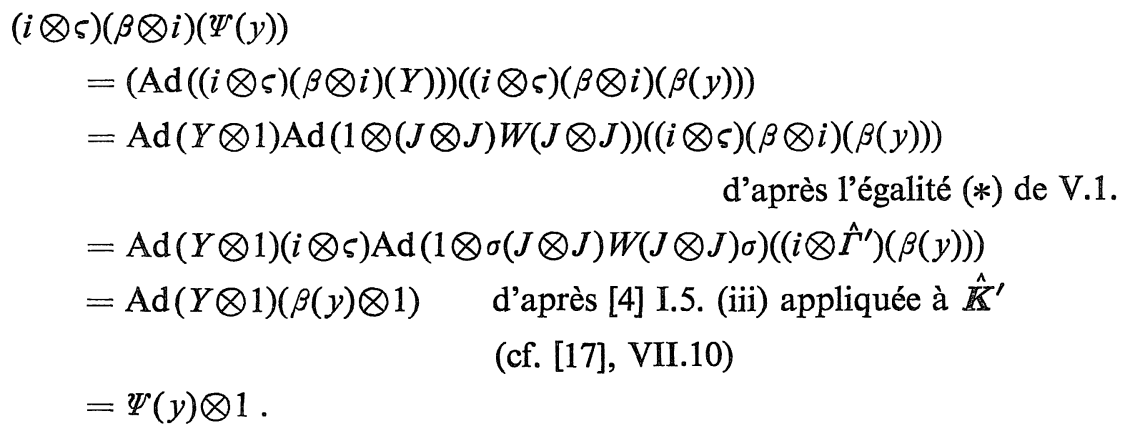

Donc $\Psi(y)$ appartient à $(B \otimes \mathcal{L}(H))^{(i \otimes s)(\beta \otimes i)}=B^{\beta} \otimes \mathcal{L}(H)$ d'après II.17. D'autre part, comme $\beta(y)$ appartient à $\mathcal{L}(\mathscr{H}) \otimes \hat{M}^{\prime}$ on obtient:

$$
\begin{aligned}
\Psi(y) & \in\left(B^{\beta} \otimes \mathcal{L}(H)\right) \cap(\text { Ad } Y)\left(\mathcal{L}(\mathcal{H}) \otimes \hat{M}^{\prime}\right) \\
& =\mathscr{S}^{*}(\delta) \quad \text { d'après IV.6. }
\end{aligned}
$$

donc $\Psi(B)=\mathscr{W}^{*}(\delta) . \quad(* *)$

Il en résulte que $C=B^{\beta}$ et $\gamma=\delta$ satisfont (i).

Proposition $V_{0}$ 3: On suppose vérifiées les conditions équivalentes de V.2..

En notant $\phi$ l'isomorphisme de $B$ sur $\mathscr{W} *(r)$, on $a$ :

(i) $\tilde{\gamma} \approx \beta(\phi, i)$

(ii) $\phi \nu(x)=1 \otimes x \quad\left(\forall x \in \hat{M}^{\prime}\right)$

De plus toute action $\gamma$ vérifiant $(i)$ et (ii) est unique à équivalence forte pres.

Démonstration: Soit $y$ dans $B$. On a, en reprenant les notations de V.2. et en notant encore $\Psi$ l'isomorphisme de $B$ sur $\mathscr{W}^{*}(\delta)$ obtenu à partir de $\Psi$ : 


$$
\begin{aligned}
\tilde{\delta}(\Psi(y)) & =\widetilde{\delta}(\operatorname{Ad} Y) \beta(y) \\
& =\operatorname{Ad}\left(1 \otimes(J J \otimes J J) W^{*}(J J \otimes J J)\right) \operatorname{Ad}(Y \otimes 1)(\beta(y) \otimes 1) \\
& \text { d'après [4], II.6 (i) } \\
& =\operatorname{Ad}(Y \otimes 1) \operatorname{Ad}\left(1 \otimes(J J \otimes J J) W^{*}(J J \otimes J J)\right)(\beta(y) \otimes 1)
\end{aligned}
$$

car d'après [5], 2.1.5. (b), $1 \otimes(J J \otimes J J) W^{*}(J J \otimes J J)$ appartient à $\mathbb{C}_{\mathcal{G l}} \otimes M^{\prime} \otimes \hat{M}^{\prime}$ et $Y \otimes 1$ appartient à $B \otimes M \otimes C_{H}$.

D'autre part, d'après [4], I.5. (ii) appliqué à $\hat{\mathbb{K}}^{\prime}$ (cf. [17], VII.10), on a:

$$
\begin{aligned}
\operatorname{Ad}\left(1 \otimes(J J \otimes J J) W^{*}(J J \otimes J J)\right)(\beta(y) \otimes 1) & =\left(i \otimes \hat{\Gamma}^{\prime}\right)(\beta(y)) \\
& =(\beta \otimes i)(\beta(y))
\end{aligned}
$$

donc:

$$
\begin{aligned}
\tilde{\delta}(\Psi(y)) & =\operatorname{Ad}(Y \otimes 1)(\beta \otimes i)(\beta(y)) \\
& =(\Psi \otimes i)(\beta(y)), \quad \text { d'où (i). }
\end{aligned}
$$

Soit $x$ dans $\hat{M}^{\prime}$. On a:

$$
\begin{aligned}
\Psi \nu(x) & =(\operatorname{Ad} Y) \beta \nu(x) \\
& =(\operatorname{Ad} Y)(\nu \otimes i) \hat{\Gamma}^{\prime}(x) \\
& =(\nu \otimes i)\left((\operatorname{Ad}(\sigma(J \otimes J) W(J \otimes J) \sigma))\left(\Gamma^{\prime}(x)\right)\right. \\
& =(\nu \otimes i)(1 \otimes x) \quad \text { d'après [5], 2.2.5. (b) appliqué } \hat{K}^{\prime} \\
& \quad \text { (cf. [17], VII.10) } \\
& =1 \otimes x, \quad \text { d'où (ii). }
\end{aligned}
$$

Soit $(C, \gamma, \phi)$ vérifiant (i) et (ii). D’après II.16., il résulte de (i) que:

$$
r(C)=\mathscr{W}^{*}(\gamma)^{\tilde{\gamma}}=\phi\left(B^{\beta}\right)
$$

Donc $\phi^{-1} \circ \gamma$ réalise un isomorphisme de $C$ sur $B^{\beta}$. De plus, pour $y$ dans $C$, on a:

$$
\begin{aligned}
(\Phi \otimes i) \delta \Phi^{-1} r(y) & =(\Phi \otimes i)(\operatorname{Ad} Y)\left(\Phi^{-1} \gamma(y) \otimes 1\right) \\
& =(\operatorname{Ad}(\phi \nu \otimes i)(\sigma(J \otimes J) W(J \otimes J) \sigma))(r(y) \otimes 1) \\
& =(\operatorname{Ad}(1 \otimes \sigma(J \otimes J) W(J \otimes J) \sigma))(r(y) \otimes 1) \quad \text { d'après (ii) } \\
& =(i \otimes \Gamma)(r(y)) \quad \text { d'après [4], I.5. (ii). } \\
& =(r \otimes i)(r(y))
\end{aligned}
$$

Ainsi:

$$
\delta \Phi^{-1} r(y)=\left(\Phi^{-1} \gamma \otimes i\right)(r(y))
$$


soit:

$$
\delta \approx \gamma\left(\Phi^{-1} \gamma, i\right) \quad \text { d'où le résultat. }
$$

Proposition V.4: Avec les hypothèses et notations de V.1.. On a:

(i) $B=\left(B^{\beta} \cup \nu\left(\hat{M}^{\prime}\right)\right)^{\prime \prime}$

(ii) Il existe un isomorphisme $\Xi$ de $\mathscr{W}^{*}(\beta)$ sur $B^{\beta} \otimes \mathcal{L}(H)$ tel que l'on ait $\widetilde{\beta} \sim(i \otimes \sigma)(\delta \otimes i)\left(\Xi, v, 1 \otimes \sigma W^{*} \sigma\right)$. (Ceci généralise [14], th. 8.2. et 8.4.).

Démonstration: L'assertion (i) résulte des égalités (*) et (**) de V.2., car $\Psi$ est injective.

D’après V.3. (i), il résulte de [4], II.8. que:

$$
\delta^{\sim \sim} \approx \widetilde{\beta}(\Psi \otimes i, i)
$$

Comme, d'après IV.3. il existe un isomorphisme $\Xi^{\prime}$ tel que

$$
\delta^{\sim \sim} \sim(i \otimes \varsigma)(\delta \otimes i) \quad\left(\Xi^{\prime}, v, 1 \otimes \sigma W^{*} \sigma\right) \text { (ii) résulte }
$$

de [4], 1.1.2.

\section{Poids dual sur le produit croise}

On utilisera les notations du chapitre II.

Définition VI.1: Soit $\psi$ dans $P(A)$. On pose $\tilde{\psi}=\psi \circ \alpha^{-1} \circ T_{\tilde{\alpha}}$; comme d'après II.4 (ii) et IV.2 (ii), $T_{\widetilde{\alpha}}$ est un poids opératoriel normal, semi-fini et fidèle de $\mathscr{W}^{*}(\alpha)$ sur $\mathscr{W}^{*}(\alpha)^{\tilde{\alpha}}=\alpha(A), \tilde{\psi}$ appartient à $P\left(\mathscr{W}^{*}(\alpha)\right)$. On dira que $\widetilde{\psi}$ est le poids dual de $\psi$.

Cette définition généralise [20], 4.3 et [6].

Proposition VI.2: Tout poids dual est $4^{-1}$-relativement invariant par rapport à $\tilde{\alpha}$.

Démonstration: Comme l'action $\tilde{\alpha}$ est intégrable, cela résulte de III.4.

Lemme VI.3: Soient $\psi_{1}$ et $\psi_{2}$ deux poids sur $A \Delta^{-1}$-relativement invariants par rapport à $\alpha$. Alors le poids $\Omega$ construit sur $A \otimes F_{2}$ à partir de $\psi_{1}$ et $\psi_{2}$ comme en [2], 1.2.2. est $\Delta^{-1}$-relativement invariant par rapport à $(i \otimes \varsigma)(\alpha \otimes i)$.

Démonstration: Soit $\omega$ dans $M_{*}^{+}$. Le poids sur $A \otimes M \otimes F_{2}$ construit comme en [2], 1.2.2. à partir de $\psi_{1} \otimes \omega$ et $\psi_{2} \otimes \omega$ est, d'après [11], p.5, égal à $(\Omega \otimes \omega)(i \otimes \varsigma)$. 
Soient alors $x_{i, j}(i, j=1,2)$ dans $A$ tels que l'élément $\sum_{i, j} x_{i, j} \otimes e_{i, j}$ de $A \otimes F_{2}$ soit positif. L'élément $\sum_{i, j} \alpha\left(x_{i, j}\right) \otimes e_{i, j}$ est positif et l'on a:

$$
\begin{aligned}
(\Omega \otimes \omega)(i \otimes \varsigma)\left(\sum_{i, j} \alpha\left(x_{i, j}\right) \otimes e_{i, j}\right) & =\left(\psi_{1} \otimes \omega\right)\left(\alpha\left(x_{1,1}\right)\right)+\left(\psi_{2} \otimes \omega\right)\left(\alpha\left(x_{2,2}\right)\right) \\
& =\left(\psi_{1}\left(x_{1,1}\right)+\psi_{2}\left(x_{2,2}\right)\right)\left\langle\Delta^{-1}, \omega\right\rangle \\
& \text { d'après III.1 (i) } \\
& =\Omega\left(\sum_{i, j} x_{i, j} \otimes e_{i, j}\right)\left\langle\Delta^{-1}, \omega\right\rangle
\end{aligned}
$$

autrement dit

$$
(\Omega \otimes i)(i \otimes \varsigma)(\alpha \otimes i)(x)=\Omega(x) \Delta^{-1} \quad\left(\forall x \in\left(A \otimes F_{2}\right)^{+}\right)
$$

d'où (i) de IIII.1.

Soient $x_{i, j}$ et $y_{i, j}(i, j=1,2)$ dans $A$ tels que

$$
x=\sum_{i, j} x_{i, j} \otimes e_{i, j} \quad \text { et } \quad y=\sum_{i, j} y_{i, j} \otimes e_{i, j}
$$

appartiennent à $\mathfrak{N}_{\mathcal{Q}}$, soit $x_{i, j}$ et $y_{i, j}$ appartiennent à $\mathfrak{R}_{\varphi_{j}}$. On a:

$$
\begin{aligned}
\left(y^{*} \otimes i\right)(i \otimes \varsigma)(\alpha \otimes i)(x) & =\left(\sum_{k, l} y_{k, l}^{*} \otimes e_{l, k} \otimes 1\right)(i \otimes \varsigma)(\alpha \otimes i)\left(\sum_{k, l} x_{i, j} \otimes e_{i, j}\right) \\
& =(i \otimes \varsigma) \sum_{i, j, k, l}\left(y_{k_{0} l}^{*} \otimes 1 \otimes e_{l, k}\right)\left(\alpha\left(x_{i, j}\right) \otimes e_{i, j}\right) \\
& =(i \otimes \varsigma) \sum_{i, j, l}\left(\left(y_{i, l}^{*} \otimes 1\right) \alpha\left(x_{i, j}\right)\right) \otimes e_{l, j}
\end{aligned}
$$

De même:

$$
(i \otimes \varsigma)(\alpha \otimes i)\left(y^{*}\right)(x \otimes 1)=(i \otimes \varsigma) \sum_{i, j, l} \alpha\left(y_{i, l}^{*}\right)\left(x_{i, j} \otimes 1\right) \otimes e_{l, j} .
$$

Ainsi, pour $\xi$ dans $\mathscr{D}\left(\Delta^{1 / 4}\right) \cap \mathscr{D}\left(\Delta^{-1 / 4}\right)$, on obtient:

$$
\begin{aligned}
& \left(\Omega \otimes \omega_{\hat{\Delta}^{1 / 4} \xi}\right)\left((i \otimes \varsigma)(\alpha \otimes i)\left(y^{*}\right)(x \otimes 1)\right) \\
& \quad=\sum_{i, j}\left(\psi_{j} \otimes \omega_{\hat{\Delta}^{1 / 4} \xi}\right)\left(\alpha\left(y_{i, j}^{*}\right)\left(x_{i, j} \otimes 1\right)\right) \\
& \quad=\sum_{i, j}\left(\psi_{j} \otimes \omega_{\hat{\Delta}^{-1 / 4}} \circ \kappa\right)\left(\left(y_{i, j}^{*} \otimes 1\right) \alpha\left(x_{i, j}\right) \quad\right. \text { d'après III.1 (ii) } \\
& \quad=\left(\Omega \otimes \omega_{\left.\hat{\Delta}^{-1 / 4} \xi^{-} \circ \kappa\right)}\right)\left(\left(y^{*} \otimes 1\right)(i \otimes \zeta)(\alpha \otimes i)(x)\right)
\end{aligned}
$$

d'où (ii) de III.1.

Proposition VI.4: Soient $\psi_{1}$ et $\psi_{2}$ deux poids sur $A \Delta^{-1}$-relativement invariants par rapport à $\alpha$. Alors, le cocycle $\left(D \psi_{1}: D \psi_{2}\right)_{t}(c f$. [2] 1.2.2.) appartient à $A^{\alpha}$ pour tout $t$ de $\mathbb{R}$.

Démonstration: Reprenons les notations de VI.3. On a: 


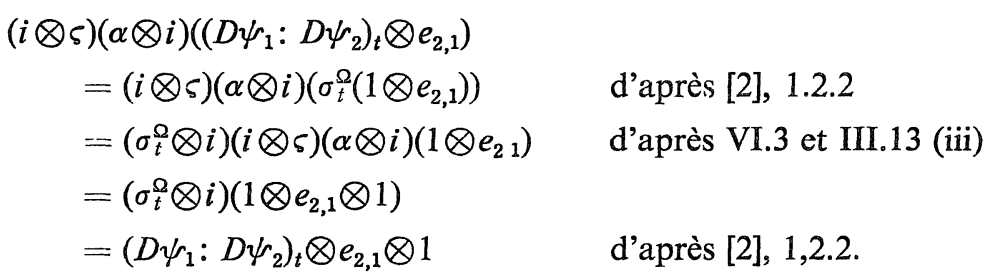

Ainsi $\left(D \psi_{1}: D \psi_{2}\right)_{t} \otimes e_{2,1}$ appartient à $\left(A \otimes F_{2}\right)^{(i \otimes s)(\alpha \otimes i)}=A^{\alpha} \otimes F_{2}$ d'après II.17, d'où le résultat.

Théorème VI.5: L'application $\psi \rightarrow \tilde{\psi}$ définie en VI.1 est une bijection de $P(A)$ sur l'ensemble des poids sur $\mathscr{W}^{*}(\alpha) \Delta^{-1}$-relativement invariants par rapport à $\widetilde{\alpha}$.

Démonstration: Soient $\psi^{\prime}$ un poids sur $\mathscr{W}^{*}(\alpha) \Delta^{-1}$-relativement invariant par rapport à $\tilde{\alpha}$ et $\psi$ dans $P(A)$. Il résulte de V1.2, de VI.4 et de IV.2. (ii) que

$$
\left(D \psi^{\prime}: D \tilde{\psi}\right)_{t} \in \mathscr{W} *(\alpha)^{\tilde{\alpha}}=\alpha(A)
$$

D'autre part d'après [9], 4.7 on a, pour tout $x$ de $A$ :

$$
\begin{aligned}
\sigma_{t}^{\psi}(\alpha(x)) & =\sigma_{t}^{\tilde{\psi}_{\circ} \omega^{-1}}(\alpha(x)) \\
& =\left(\sigma_{t}^{\psi}(x)\right) .
\end{aligned}
$$

Donc, comme $\left(D \psi^{\prime}: D \widetilde{\psi}\right)_{t}$ est un $\sigma_{t}^{\widetilde{\psi}}$-cocycle, on obtient que $\alpha^{-1}\left(\left(D \psi^{\prime}: D \widetilde{\psi}\right)_{t}\right)$ est un $\sigma_{t}^{\psi}$-cocycle. Dans ces conditions, le théorème 1.2.4 de [2] donne l'existence de $\psi_{1}$ dans $P(A)$ tel que $\left(D \psi_{1}: D \psi\right)_{t}=\alpha^{-1}\left(\left(D \psi^{\prime}: D \tilde{\psi}\right)_{t}\right)$. Soit aussi:

$$
\begin{aligned}
\left(D \tilde{\psi}^{\prime}: D \psi\right)_{t} & =\alpha\left(\left(D \psi_{1}: D \psi\right)_{t}\right) \\
& =\left(D \psi_{1} \circ \alpha^{-1}: D \psi \circ \alpha^{-1}\right)_{t} \\
& =\left(D \tilde{\psi}_{1}: D \tilde{\psi}\right)_{t} \quad \text { d'après [9], 4.7. }
\end{aligned}
$$

ce qui implique ([2], th. 1.3.4.)

$$
\psi^{\prime}=\tilde{\psi}_{1}
$$

ce qui, compte tenu de VI.2, achève la démonstration.

Cas particulier V1.6: Soit $G$ un groupe localement compact.

(i) Soit $\beta$ une action continue de $G$ sur $A$. D'après [4], II.2 (ii), le produit croisé au sens de [20], 3.3., noté $\mathscr{R}(A, \beta)$ est égal à $\mathscr{W})^{*}\left(\beta^{g}\right)$. Une théorie du poids dual sur $\mathscr{R}(A, \beta)$ est développée dans [3], [10], [16] et [20]; une nouvelle construction en est donnée dans [6]. Celle ci est en fait un cas 
particulier de la définition VI.1.

Il en résulte que l'application $\psi \rightarrow \widetilde{\psi}$ une bijection de $P(A)$ sur l'ensemble des poids $\operatorname{sur} \mathscr{R}(A, \beta)$ invariants par rapport à $\widetilde{\beta}^{g}$. (Ce résultat a été obtenu indépendamment dans [19], III.12).

(ii) Soit $\alpha$ une action de $K S(G)$ sur $A$. D'après [4], I.3 (ii), il existe une action continue $r$ de $G$ sur $\mathscr{W}^{* *}(\alpha)$ telle que $\gamma^{d}=\widetilde{\alpha}$. Il en résulte que l'application $\psi \rightarrow \tilde{\psi}$ est une bijection de $P(A)$ sur l'ensemble des poids sur $\mathscr{W}^{* *}(\alpha)$ relativement invariants par rapport à $\gamma$, de module $\Delta_{G}^{-1}$.

Ainsi, dans le cas où $G$ n'est pas unimodulaire, cette dernière affirmation fait apparaître que le théorème III.2.1 de [19] est erronné. En effet, alors le poids opératoriel $Q_{G}$ (notation de [19]) n'est pas invariant, mais relativement invariant.

\section{Bibliographie}

[1] Combes F., Poids sur une $C^{*}$-algèbre, J. Math. pures et appl., 47 (1968), 57-100.

[2] Connes A., Une classification des facteurs de type III, Ann. Sc. Ec. Norm. Sup., 4ème série, 6, fasc. 2, (1973), 133-252.

[3] Digernes T., Duality for weights on covariant systems and its applications, Dissertation, U.C.L.A., 1975.

[4] Enock M., Produit croisé d'une algèbre de von Neumann par une algèbre de Kac, J.F.A., 26 (1977), 16-47.

[5] Enock M. et Schwartz J. M., Une dualité dans les algèbres de von Neumann, Bull. Soc. Math. France, Supp. Mémoire 44 (1975), 1-144.

[6] - Une nouvelle construction du poids dual sur le produit croisé d'une algèbre de von Neumann par un groupe localement compact. C.R. Acad. Sc. Paris, série A. 282 (1976), 415-418.

[7] - Algèbres de Kac et produits croisés, C.R. Acad. Sc. Paris, série A, 283 (1976), 321-323.

[8] Haagerup U., The standard form of von Neumann algebras, K $\phi$ benhavns Univ. Math. Inst., Preprint series $15,1973$.

[9] —-, Operator valued weights in von Neumann algebras, Odense Mat. Inst. Preprint 12, 1975.

[10] $\longrightarrow$ On the dual weights for crossed products of von Neumann algebras, Odense Mat. Inst. Preprint 10, 1975.

[11] Havet J.F., Quelques propriétés des systèmes dynamiques non commutatifs, thèse 3ème cycle, Univ. Orléans, 1976.

[12] Landstad M., Duality for covariant systems. Trondheim Preprint, 1974.

[13] - Duality for dual covariance algebras, Comm. Math. Phys., 52, (1977), 191-202.

[14] Nakagami Y., Dual action on a von Neumann algebra and Takesaki's duality for a locally compact group, Publ. R.I.M.S., Kyoto Univ., 12 (1977), 727-775.

[15] Pedersen G. K. et Takesaki M., The Radon-Nikodym theorem for von Neumann algebras, Acta. Math., 130 (1973), 53-87.

[16] Sauvageot J. L., Sur le type du produit croisé d'une algèbre de von Neumann par un groupe localement compact, Bull. Soc. Math. France, à paraître 
[17] Schwartz J. M., Sur la structure des algèbres de Kac, Tirage préliminaire, 2ème édition, Novembre 1977.

[18] Strătilă, Ș. Voiculescu D. et Zsidó L., Sur les produits croisés, C.R. Acad. Sc. Paris, série $A, 282$ (1976), 779-782.

[19] - On crossed products, I et II, Rev. Roumaine Math. P. et appl. 21 (1976), 1411-1449 et 22 (1977), 83-117.

[20] Takesaki M., Duality for crossed products and the structure of von Neumann algebras of type III. Acta. Math., 131 (1973), 249-310.

[21] Tomiyama J., On the projections of norm one in $W^{*}$-algebras, Proc. Jap. Acad. 33, (1957), 608-612. 